\title{
Budget \\ FY 1995 Site Support Program Plan WBS 6.10.1
}

\author{
G. M. Wemhoff \\ Date Published \\ September 1994
}

Prepared for the U.S. Department of Energy Office of Environmental Restoration and Waste Management

\section{(29) Westinghouse




\section{DISCLAIMER}

This report was prepared as an account of work sponsored by an agency of the United States Government. Neither the United States Government nor any agency thereof, nor any of their employees, make any warranty, express or implied, or assumes any legal liability or responsibility for the accuracy, completeness, or usefulness of any information, apparatus, product, or process disclosed, or represents that its use would not infringe privately owned rights. Reference herein to any specific commercial product, process, or service by trade name, trademark, manufacturer, or otherwise does not necessarily constitute or imply its endorsement, recommendation, or favoring by the United States Government or any agency thereof. The views and opinions of authors expressed herein do not necessarily state or reflect those of the United States Government or any agency thereof. 


\section{DISCLAIMER}

Portions of this document may be illegible in electronic image products. Images are produced from the best available original document. 


\section{RELEASE AUTHORIZATION}

Document Number: $\quad$ WhC-SP-1109

Document Title: BUDGET FY 1995 SITE SUPPORT PROGRAM PLAN WBS 6.10.1

Release Date: $\quad 9 / 22 / 94$

$* * * * * * * * * * * *$

This document was reviewed following the procedures described in WHC-CM-3-4 and is:

APPROVED FOR PUBLIC RELEASE

$* * * * * * * * * * * * *$

WHC Information Release Administration Specialist:

Chisterw Itillixgtears

C. Hillingham

$9 / 22 / 94$

(Signature)

(Date) 


\section{Site Support Program Plan Approval Sheet}

$\frac{6.10 .1}{\text { BUDGET }}$

Assistant Manager-Contracting Officer's Representative
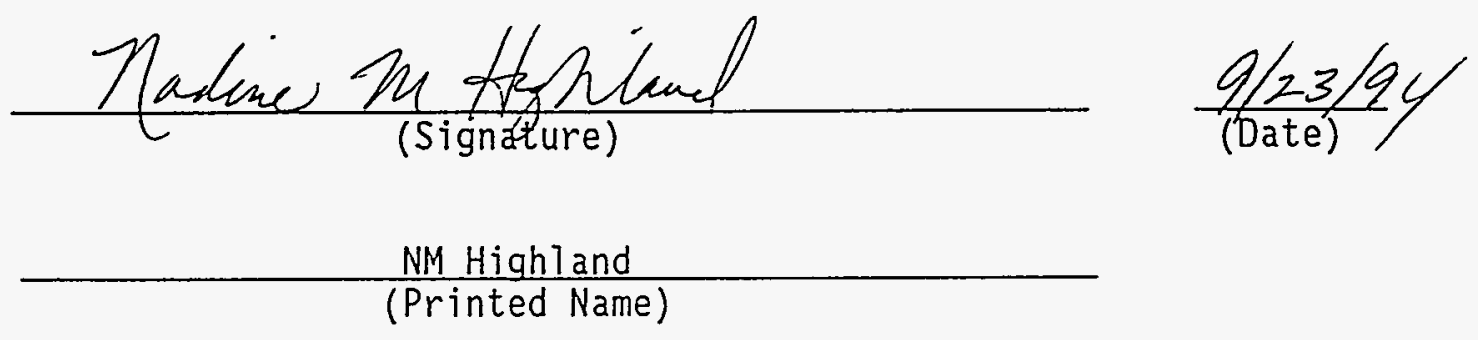

$\frac{\text { NM Highland }}{\text { (Printed Name) }}$

\section{RL Program Manager}
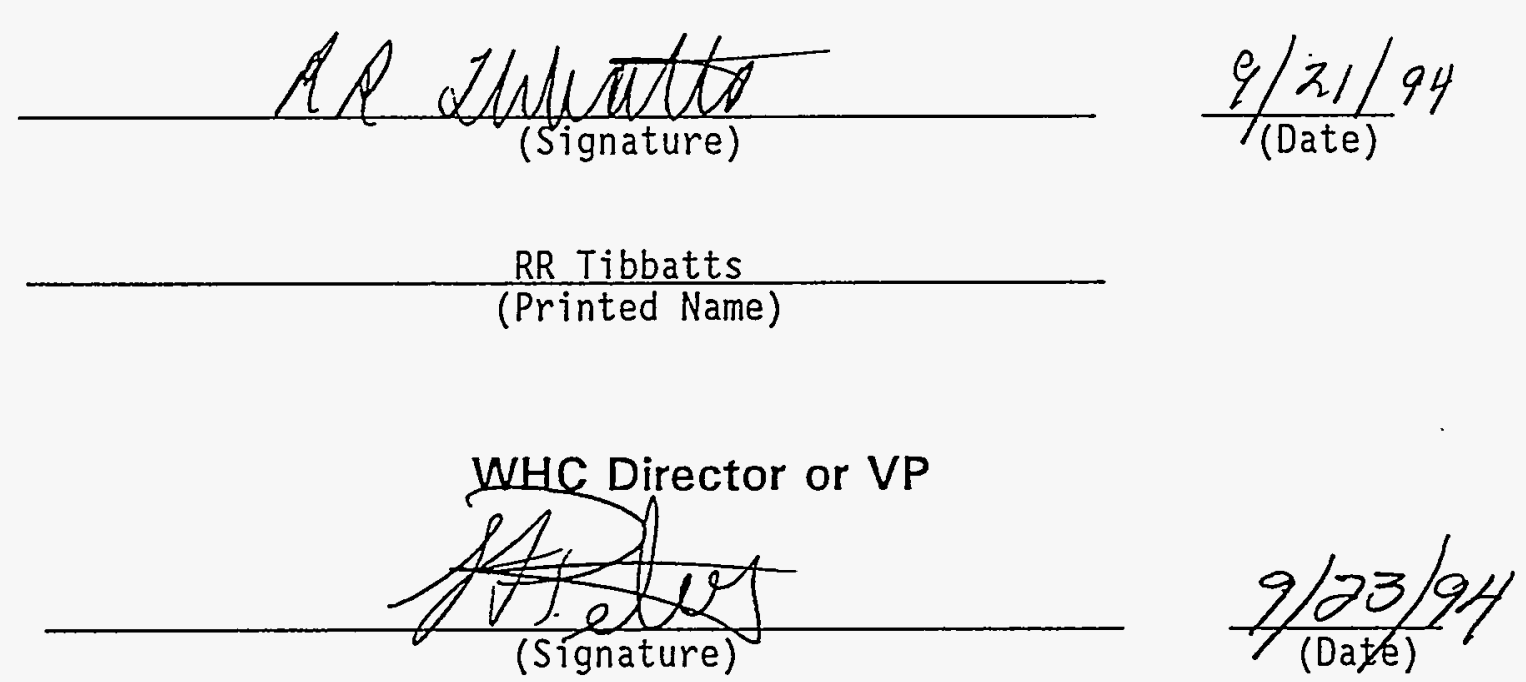

LF Peters

(Printed Name)

WHC Program Manager

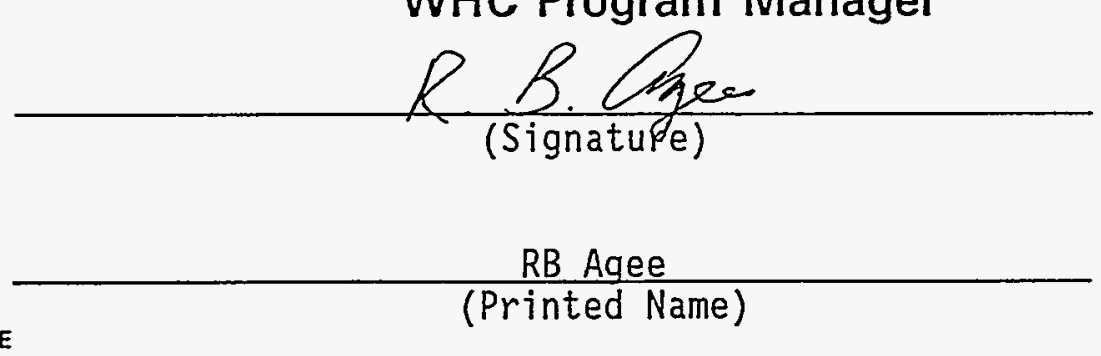




\begin{tabular}{|c|c|c|}
\hline Index & $\begin{array}{c}\text { Westinghouse Hanford Company } \\
\text { Finance and Administration } \\
\text { SMS/WBS 6.10 }\end{array}$ & $\begin{array}{c}\text { FY 1995 } \\
\text { Site Support Program Plan } \\
\text { August 31, 1994 }\end{array}$ \\
\hline
\end{tabular}

\section{BUDGET \\ WBS 6.10.1}

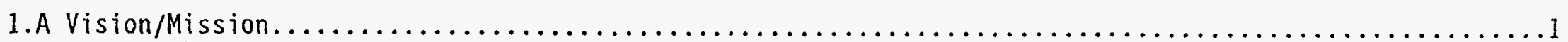

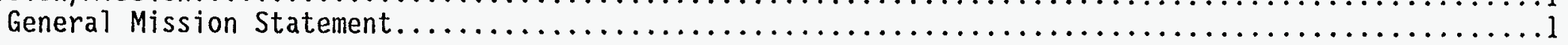

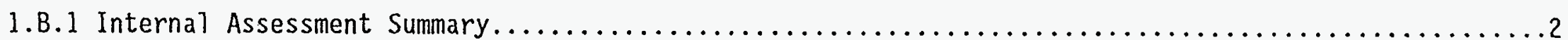

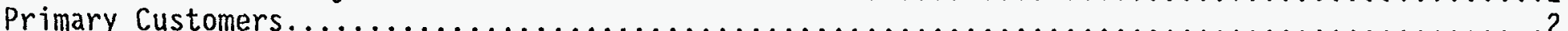

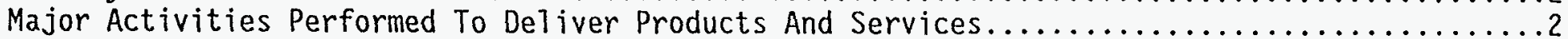

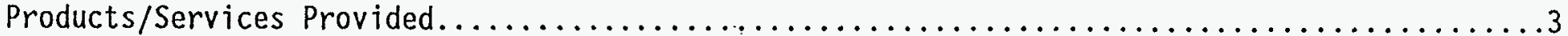

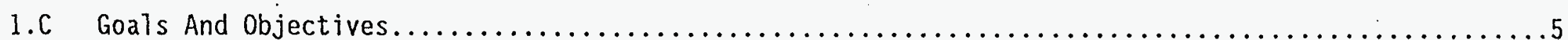

Program Priorities.........

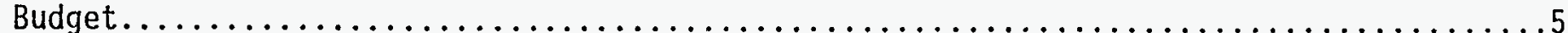

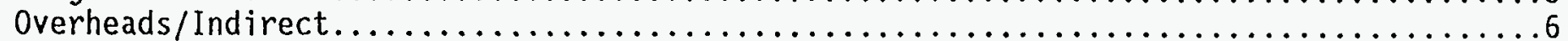

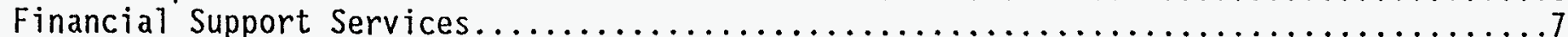

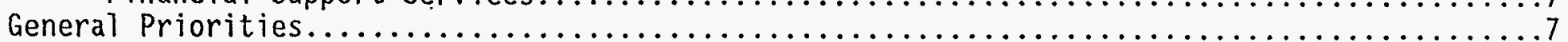

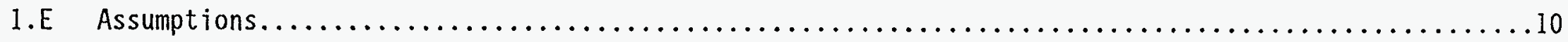

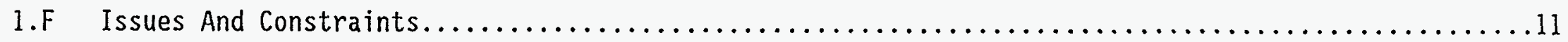

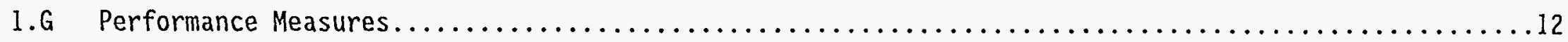

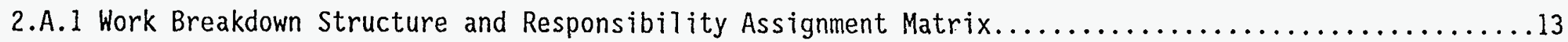

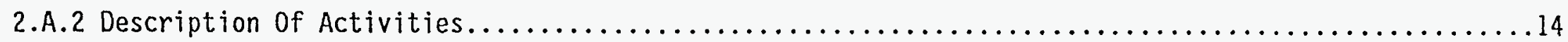

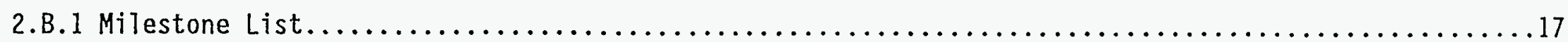




\begin{tabular}{|c|c|c|}
\hline Index & $\begin{array}{c}\text { Westinghouse Hanford Company } \\
\text { Finance and Administration } \\
\text { SMS/WBS 6.10 }\end{array}$ & $\begin{array}{c}\text { FY 1995 } \\
\text { Site Support Program Plan } \\
\text { August 31, 1994 }\end{array}$ \\
BUDGET \\
WBS 6.10.1
\end{tabular}

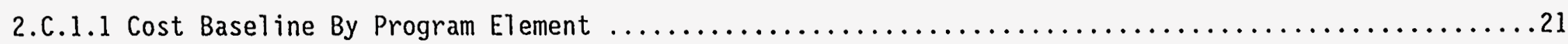

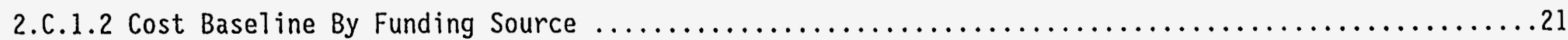

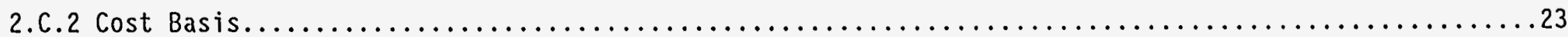

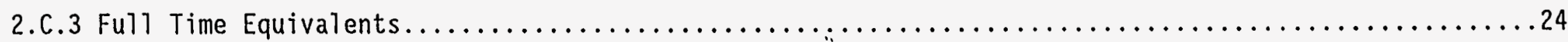

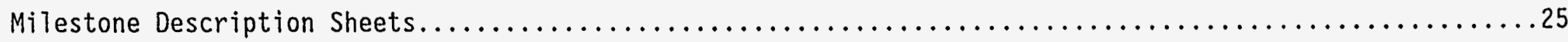




\begin{tabular}{|c|c|c|}
\hline 1.A Vision/Mission & $\begin{array}{c}\text { Westinghouse Hanford Company } \\
\text { Finance and Administration } \\
\text { SMS/WBS 6.10 }\end{array}$ & $\begin{array}{c}\text { FY 1995 } \\
\text { Site Support Program Plan } \\
\text { June 30, 1994 }\end{array}$ \\
BUDGET \\
WBS 6.10.1
\end{tabular}

\section{GENERAL MISSION STATEMENT}

The mission of the Budget function includes directing and monitoring the formulation, execution, and control of all program and indirect budgets. Additionally, Budget is responsible for the implementation of budgetary controls on all funds obligated to WHC. 


\begin{tabular}{|c|c|c|}
\hline 1.B.1 Internal Assessment Summary & $\begin{array}{c}\text { Westinghouse Hanford Company } \\
\text { Finance and Administration } \\
\text { SMS/WBS } 6.10\end{array}$ & $\begin{array}{c}\text { FY } 1995 \\
\text { Site Support Program Plan }\end{array}$ \\
BUDG.ET \\
WBS 6.10.1
\end{tabular}

\section{PRIMARY CUSTOMERS}

The Department of Energy, Richland Operations Office (RL) Budget Division

Westinghouse Hanford Company Financial/Cost Account Managers

Westinghouse Hanford Company Programs

Westinghouse. Electric Corporation

DOE-RL/WHC Chief Financial officers

\section{MAJOR ACTIVITIES PERFORMED TO DELIVER PRODUCTS AND SERVICES}

Conduct Field Audits of Internal Financial Controls

Consolidate Field Input to Execution Year Financial Exercises/Requests (Direct \& Indirect)

Coordinate Indirect (Scope \& Funding) Budget and Direct Budget Non-EM Pricing Validation Reviews

Coordinate Preparation and Maintenance of the WHC Budget Manual

Coordination of Analysis of WHC Uncosted Obligations

Interface with Other Hanford Contractor Financial Personnel

Maintain/Prepare WHC Staffing Reports/Status 


\begin{tabular}{|l|c|c|}
\hline 1.B.1 Internal Assessment Summary & $\begin{array}{c}\text { Hestinghouse Hanford Company } \\
\text { Finance and Administration } \\
\text { SMS/WBS 6.10 }\end{array}$ & $\begin{array}{c}\text { FY 1995 } \\
\text { Site Support Program Plan }\end{array}$ \\
\hline
\end{tabular}

\section{BUDGET \\ WBS 6.10.1}

MAJOR ACTIVITIES PERFORMED TO DELIVER PRODUCTS AND SERVICES (continued)

Preparation/Issuance of Guidance Letters (Unified Budget Ca77, Spending Targets, Current \& Outyear Rates, FWP's, etc..)

Prepare SMS Executive Summary Charts

Present Financial Overviews to WHC/DOE-RL CFO's (\& Senior Staff)

Regular Interface With WHC Financial Organization (Community)

Update/Maintain Funds Control Table

Indirect Review Board

Distribution of Indirect Pool Variances

Summary Analysis of Indirect Cost And Liquidation Trends

Management of Indirect Baseline

PRODUCTS/SERVICES PROVIDED

Coordinate/Administer Financial Reporting on DOE-HQ ADS's

Coordination of WHC Unified Budget Call Budget Submission 


\begin{tabular}{|c|c|c|}
\hline 1.B.I Internal Assessment Summary & $\begin{array}{c}\text { Westinghouse Hanford Company } \\
\text { Finance and Administration } \\
\text { SMS/WBS } 6.10\end{array}$ & $\begin{array}{c}\text { FY } 1995 \\
\text { Site Support Program Plan }\end{array}$ \\
BUDGET \\
WBS 6.10 .1
\end{tabular}

PRODUCTS/SERVICES PROVIDED (continued)

Overhead Planning Coordination

SMS Reporting/SSPP Development

WHC Budget Mạual

WHC Funds Reconciliation

HQ-ADS Executive Summary

Al Tocable Cost Summary

Unmatched/Undistributed Cost Summary

Quarterly Surveillances of WHC Internal Financial Controls

Miscellaneous Reports

WN/ZN Quarterly Report

Biweek7y DOE-RL CFO Status Report

- To/From Other Hanford Contractors

ir Analysis of Funding Base for Award Fee

\author{
Corporate Financial Reporting \\ Special Requests \\ WHC Financial Status Briefings \\ Indirect Rates And Assessments Manual \\ Inter-Contractor Financial Coordination \\ Indirect Financial (Services \& Overheads) Summary
}

FIVRS

- Staffing

- Drawdown Projections 


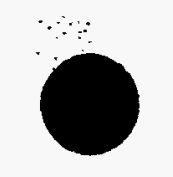

\begin{tabular}{|l|c|c|}
\hline $1 . C$ Goals and Objectives & $\begin{array}{c}\text { Westinghouse Hanford Company } \\
\text { Finance and Administration } \\
\text { SMS/WBS 6.10 }\end{array}$ & $\begin{array}{c}\text { FY } 1995 \\
\text { Site Support Program P1an }\end{array}$ \\
\hline
\end{tabular}

\section{BUDGET \\ WBS 6.10.1}

\section{PROGRAM PRIORITIES}

\section{BUDGET}

1. Coordinate and integrate all program (EM, excluding ADS submission, and non-EM) budget submissions and related special company level exercises to ensure compliance with financial and budgetary regulations.

2. Maintain company-level tracking of all current year programmatic funding, fiscal year spending forecasts, commitments and year-end trends (i.e., trends during the year as well as year-to-year comparison).

3. Ensure timely commitment and monitoring of Capital Equipment, Small Projects, and General Plant Project funds.

4. Development and maintenance of an approved budget manual that includes documentation standards.

5. Conduct independent analysis to assure proper documentation and adequate justification exists to support budget requests to allow for independent and/or customer validation.

6. Conduct periodic independent analysis of program uncosted obligations for budget formulation certification to ensure balances are accurately reflected and are considered in the development of requests for new budget authority. 


\begin{tabular}{|l|c|c|}
\hline 1.C Goals and Objectives & $\begin{array}{c}\text { Westinghouse Hanford Company } \\
\text { Finance and Administration } \\
\text { SMS/WBS } 6.10\end{array}$ & Site Support Program PIan \\
\hline
\end{tabular}

\section{BUDGET \\ WBS 6.10.1}

\section{PROGRAM PRIORITIES}

\section{OVERHEADS/INDIRECT}

1. Maintain company-7evel tracking of all current year indirect funding, fiscal year spending forecasts, Tiquidations, and cost trends.

2. Provide financial planning processes and oversee the development of overhead/indirect budgets to ensure they support programmatic missions and requirements.

3. Monitor and manage the authorized spending levels of company-level overhead to ensure DOE issued ceilings are not exceeded.

4. Coordinate review/analysis of direct versus indirect funding determinations.

5. Monitor overhead/indirect change requests and provide assessment of impact to DOE overhead funding $\operatorname{target}(s)$, rate(s), and ECCEL.

6. Provide assessment of impact to Programs resulting from overhead/indirect rate changes and/or distribution of variances.

7. Submit indirect liquidation rates to RL for approval.

8. Request changes in company-level overhead and service pool liquidation methodologies, if appropriate. 



\begin{tabular}{|l|c|c|}
\hline I.C Goals and Objectives & $\begin{array}{c}\text { Westinghouse Hanford Company } \\
\text { Finance and Administration } \\
\text { SMS/WBS 6.10 }\end{array}$ & $\begin{array}{c}\text { FY 1995 } \\
\text { Site Support Program Plan }\end{array}$ \\
\hline
\end{tabular}

\section{BUDGET \\ WBS 6.10.1}

\section{GENERAL PRIORITIES (continued)}

5. Provide oversight/coordination of all WHC budgetary activities.

6. Coordinate miscellaneous budget schedules and crosscuts as required in the annual Unified Budget Cal1 issued by RL Budget Division.

7. Develop and maintain company-level funding profiles; and prepare responses involving budgetary data (e.g., funding and manpower) as requested by RL Management, DOE-HQ, OMB, Congress, the - Media, and/or the public. This includes coordinating manpower projections with Human Resources.

8. Develop/coordinate the preparation of budget briefings, especially indirect, to $\mathrm{RL} / \mathrm{HQ} /$ State/EPA/etc. .

9. Preparation of other miscellaneous requirements (e.g., Outlays, FIVRS, Reimbursable Work, Allocable Cost, Overhead/Indirect activity pool status, monthiy SMS reporting, manpower analysis and reporting, CFO biweekiy, and AFP/DISCAS/SMS reconciliation).

10. Maintain funding controts to ensure effective startup, mid-year review, and closeout of each fiscal year.

12. Ensure that all staff responsible for preparing or reviewing program and indirect budgets are trained to adhere to proper financial and budgetary standards.

13. Evaluate the effectiveness of the internal program budget development process to ensure that well reasoned, defendable budgets are being produced. 


\begin{tabular}{|l|c|c|}
\hline I.C Goals and Objectives & $\begin{array}{c}\text { Westinghouse Hanford Company } \\
\text { Finance and Administration } \\
\text { SMS/WBS } 6.10\end{array}$ & $\begin{array}{c}\text { FY 1995 } \\
\text { Site Support Program Plan }\end{array}$ \\
\hline
\end{tabular}

\section{BUDGET WBS 6.10.1}

\section{GENERAL PRIORITIES (Continued)}

14. Perform activities to ensure effective utilization, proper control and execution of all obligated funds, including monitoring fiscal year spending forecasts.

15. Provide adequate oversight through surveillances and reviews, and internal audits based on an analysis of audit risks. 


\begin{tabular}{|l|c|c|}
\hline I.E Assumptions & $\begin{array}{c}\text { Westinghouse Hanford Company } \\
\text { Finance and Administration } \\
\text { SMS/WBS 6.10 }\end{array}$ & $\begin{array}{c}\text { FY 1995 } \\
\text { Site Support Program Plan }\end{array}$ \\
\hline
\end{tabular}

\section{BUDGET WBS 6.10.1}

\section{ASSUMPTIONS}

- Budget schedules for EM Programs must be consistent with requirements identified in the ADS submission, as modified by subsequent change requests.

- Budget submissions for non-EM only programs must be consistent with the Unified Budget Call and associated program guidance.

- Program budget funding baselines should be consistent with FY funding levels as identified in guidance received from RL Budget Division, including productivity challenges. Proposed supplemental/reprogramming requests to cover identified shortfalls should not be assumed in developing the program budget funding baselines.

- Coordination of activities between Financial Integration \& Planning (6.10.1.1) and Program Baseline Integration (1.8.2) is assumed. 


\begin{tabular}{|c|c|c|}
\hline 1.F Issues and Constraints & $\begin{array}{c}\text { Westinghouse Hanford Company } \\
\text { Finance and Administration } \\
\text { SMS/WBS 6.10 }\end{array}$ & $\begin{array}{c}\text { FY 1995 } \\
\text { Site Support Program P1an }\end{array}$ \\
\hline BUDGET \\
WBS 6.10.1
\end{tabular}

\section{ISSUES AND CONSTRAINTS}

- The ADS submission is excluded from the requirements set forth in this WBS unless otherwise noted.

- Activities must comply with current applicable regulations and guidance:

- Federal/State/etc. Regulations

$\quad$ WHC M\&O Contract

- DOE Orders and Directives

- DOE/RL Guidance/Correspondence

- Labor Contracts

- WHC Management Guidance

- Management Requirements and Procedures (MRP), Management Policies and Other Controlled Manuals. 


\begin{tabular}{|c|c|c|}
\hline 1.G Performance Measures & $\begin{array}{c}\text { Westinghouse Hanford Company } \\
\text { Finance and Administration } \\
\text { SMS/HBS 6.10 }\end{array}$ & $\begin{array}{c}\text { FY 1995 } \\
\text { Site Support Program PIan }\end{array}$ \\
\hline BUDGET \\
WBS 6.10.1
\end{tabular}

Based upon the level of effort required to complete activities, performance measures can only be defined by the specific milestones and/or special requests completed on schedule or as requested. 


\begin{tabular}{|l|c|c|}
\hline $\begin{array}{l}\text { Work Breakdown } \\
\text { Structure }\end{array}$ & $\begin{array}{c}\text { Westinghouse Hanford Company } \\
\text { Finance and Administration } \\
\text { SMS/WBS No. 6.10 }\end{array}$ & $\begin{array}{c}\text { FY 1995 } \\
\text { Site Support Program Plan }\end{array}$ \\
\hline
\end{tabular}

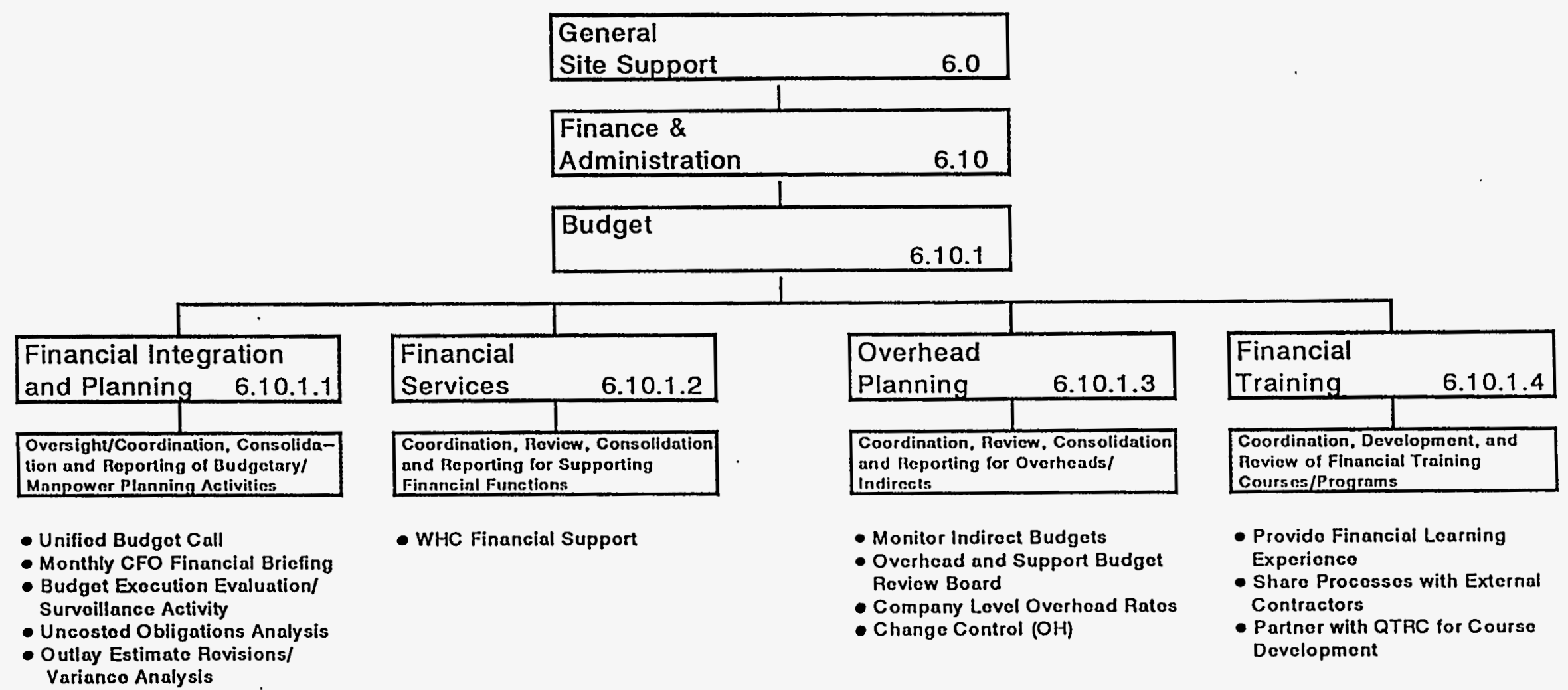




\begin{tabular}{|c|c|c|c|c|c|}
\hline \multicolumn{2}{|c|}{$\begin{array}{l}\text { 2.A.1 } \\
\text { Work Breakdown Structure and } \\
\text { Responsibility Assignment Matrix }\end{array}$} & \multicolumn{2}{|c|}{$\begin{array}{c}\text { Westinghouse Hanford Company } \\
\text { Finance and Administration } \\
\text { SMS/WBS } 6.10\end{array}$} & \multicolumn{2}{|c|}{$\begin{array}{c}\text { FY } 1995 \\
\text { Site Support Program Plan }\end{array}$} \\
\hline Program Element & $\begin{array}{c}\text { Activity/Program } \\
\text { Sub-Element }\end{array}$ & Cost Account & Title & $\begin{array}{l}\text { Responsible } \\
\text { Manager }\end{array}$ & $\begin{array}{l}\text { Responsible } \\
\text { Organization }\end{array}$ \\
\hline 6.10 & & IMDDI6 & $\begin{array}{l}\text { Finance and } \\
\text { Administration }\end{array}$ & EP Vodney & $\begin{array}{l}\text { Financial Admin } \\
\& \text { Control }\end{array}$ \\
\hline 6.10 .1 & 6.10 .1 .1 & 1MDD1600 & Budget & EP Vodney & $\begin{array}{l}\text { Financial Admin } \\
\& \text { Control }\end{array}$ \\
\hline 6.10 .1 & 6.10 .1 .1 & $1 M D D 160000$ & $\begin{array}{l}\text { Financial } \\
\text { Integration \& } \\
\text { Planning }\end{array}$ & RB Agee & $\begin{array}{l}\text { Financial Admin } \\
\text { \& Control }\end{array}$ \\
\hline 6.10 .1 & 6.10 .1 .2 & IMDD1601 & $\begin{array}{l}\text { Financial Control } \\
\text { \& Analysis } \\
\end{array}$ & LH Peterson & $\begin{array}{l}\text { Financial Admin } \\
\text { \& Control }\end{array}$ \\
\hline 6.10 .1 & 6.10 .1 .2 & $1 M D D 160100$ & $\begin{array}{l}\text { Financial } \\
\text { Services }\end{array}$ & LC Brandt & $\begin{array}{l}\text { Financial Admin } \\
\text { \& Control }\end{array}$ \\
\hline 6.10 .1 & 6.10 .1 .3 & IMDD160200 & Overhead Planning & BR Thomas & $\begin{array}{l}\text { Financial Admin } \\
\text { \& Control }\end{array}$ \\
\hline 6.10 .1 & 6.10 .1 .3 & 1MDD160201 & Business Rotation & BR Thomas & $\begin{array}{l}\text { Financial Admin } \\
\text { \& Control } \\
\end{array}$ \\
\hline 6.10 .1 & 6.10 .1 .4 & $1 M D 0160300$ & $\begin{array}{l}\text { Financial } \\
\text { Training }\end{array}$ & RB Agee & $\begin{array}{l}\text { Financial Admin } \\
\text { \& Control }\end{array}$ \\
\hline & & & & & \\
\hline
\end{tabular}




\begin{tabular}{|c|c|}
\hline 2.A.2 Description of Activities & $\begin{array}{c}\text { Hestinghouse Hanford Company } \\
\text { Finance and Administration } \\
\text { SMS/WBS } 6.10\end{array}$ \\
\hline ACTIVITY & DESCRIPTION \\
\hline $\begin{array}{l}\text { Site Management System Monthly } \\
\text { Report } 6.10 .1\end{array}$ & $\begin{array}{l}\text { The activity includes delivery of a monthly SMS report which } \\
\text { includes: Program Executive Summary; Program Manager's Assessment; } \\
\text { Milestone Schedule, Achievements, and Exception reports; Program } \\
\text { Accomplishment; Change Request Status; Expense Cost Performance and } \\
\text { Analysis; and an Issues section. }\end{array}$ \\
\hline FY 1996 Site Support Program Plan & $\begin{array}{l}\text { The activity includes preparation and submittal of the FY } 1996 \\
\text { program plan for } 6.10 .1 \text { Budget to include: A Vision/Mission } \\
\text { statement; Situational Analysis; Goals and Objectives; Strategies; } \\
\text { Assumptions; Issues and Constraints; Performance Measures; } \\
\text { Technical, Schedule and Cost Baselines; and Execution Year } \\
\text { Milestone descriptions. }\end{array}$ \\
\hline Unified Budget Call & $\begin{array}{l}\text { Coordinate and prepare Program budget submissions for non-EM in the } \\
\text { format, detail, and schedule as outlined in the Annual Unified } \\
\text { Budget Call issued by RL. }\end{array}$ \\
\hline Budget Manual & $\begin{array}{l}\text { Coordinate, develop and maintain an approved budget manual that } \\
\text { includes documentation standards. Merging of existing manuals with } \\
\text { possible title changes is acceptable. }\end{array}$ \\
\hline $\begin{array}{l}\text { Systematic Uncosted Obligations } \\
\text { Analyses }\end{array}$ & $\begin{array}{l}\text { Coordinate annual and interim reports that includes projections be } \\
\text { made of the upcoming months and fiscal year end, as well as } \\
\text { reporting the uncosted actuals to date. The annual report will } \\
\text { also need to include projections for the next fiscal year. } \\
\text { Narrative is required for both the annual and the interims; on all } \\
\text { balances by ADS for the annual and on all balances over } \$ 1 M \text { by } \\
\text { program for the interims. }\end{array}$ \\
\hline $\begin{array}{l}\text { Internal Evaluation/Surveillance } \\
\text { Schedule, Reports, and Corrective } \\
\text { Action Plans }\end{array}$ & $\begin{array}{l}\text { Content of reports to include area(s) evaluated, reviews completed, } \\
\text { results of reviews, and corrective action plans. Coordinate with } \\
\text { Planning Systems Integration/Planning and Mgmt Control Systems. }\end{array}$ \\
\hline
\end{tabular}




\begin{tabular}{|c|c|}
\hline 2.A.2 Description of Activities & $\begin{array}{l}\text { Westinghouse Hanford Company } \\
\text { Finance and Administration } \\
\text { SMS/WBS } 6.10\end{array}$ \\
\hline ACTIVITY & DESCRIPTION \\
\hline $\begin{array}{l}\text { Outlay Projections and Variance } \\
\text { Reporting }\end{array}$ & $\begin{array}{l}\text { Coordinate and develop outlay revisions to the FY } 1995 \text { outlay } \\
\text { estimates by appropriation. Provide RL with acceptable narrative } \\
\text { explanations of outlay variances (actual versus estimate) as } \\
\text { requested by RL. RL will provide guidance as to what } \\
\text { appropriations require narrative by the 8th working day of the } \\
\text { month due. }\end{array}$ \\
\hline Administrative Control of Funds & Monthly update/maintain Funds Control Tables in FDS. \\
\hline $\begin{array}{l}\text { Financial Information Variance } \\
\text { Reporting System (FIVRS) Cost } \\
\text { Estimate }\end{array}$ & $\begin{array}{l}\text { FIVRS Cost Estimating provides DOE-RL with monthly cost projections } \\
\text { for Defense Program (DP) operating dollars and associated monthly } \\
\text { narrative explanations when variances to cost projections are } \\
\text { generated. FIVRS reporting occurs semi-annually. }\end{array}$ \\
\hline $\begin{array}{l}\text { Transfers To/From Other Hanford } \\
\text { Contractors }\end{array}$ & $\begin{array}{l}\text { The purpose of the To/From Other Hanford Contractors is to provide } \\
\text { RL with a projection of funding required to accomplish various work } \\
\text { scope between contractors. WHC provides projections for transfers } \\
\text { to and from PNL, HEHF, KEH, and RL. KEH does not submit a separate } \\
\text { report, their projection is provided on the WHC submittal. }\end{array}$ \\
\hline $\begin{array}{l}\text { Monthly Budget Briefing Report to } \\
\text { RL-CFO }\end{array}$ & $\begin{array}{l}\text { The monthiy budget briefing to RL's CFO is an overall company } \\
\text { financial briefing. The briefing includes commitments of capital } \\
\text { equipment and GPP as well as a fiscal year spending forecast } \\
\text { analyses by program. }\end{array}$ \\
\hline $\begin{array}{l}\text { Transition of Training Courses to } \\
\text { the Quality Training Resource } \\
\text { Center (QTRC) }\end{array}$ & $\begin{array}{l}\text { Scope includes transfer of administration and instructional design } \\
\text { support to QTRC. Provide line management ownership of content/ } \\
\text { subject and coordination of competent training volunteers. }\end{array}$ \\
\hline $\begin{array}{l}\text { Overhead Planning Self-Surveillance } \\
\text { for Improvements to the FY } 1995 \\
\text { Indirect Planning Process }\end{array}$ & $\begin{array}{l}\text { RL issues an overhead funding target prior to the start of the } \\
\text { fiscal year of which WHC indirect programs are included. WHC is to } \\
\text { reconcile the issued target to the indirect budget requests. }\end{array}$ \\
\hline
\end{tabular}




\begin{tabular}{|c|c|}
\hline 2.A.2 Description of Activities & $\begin{array}{c}\text { Hestinghouse Hanford Company } \\
\text { Finance and Administration } \\
\text { SMS/WBS } 6.10\end{array}$ \\
\hline ACTIVITY & DESCRIPTION \\
\hline $\begin{array}{l}\text { Provide quarterly SAS Obligation } \\
\text { and cost report }\end{array}$ & $\begin{array}{l}\text { Report provides a status of SAS activities against "planned } \\
\text { estimates". The FY } 1995 \text { SAS Budget Estimates for operating } \\
\text { expense, capital equipment, general plant projects, and line item } \\
\text { construction projects as submitted are used. }\end{array}$ \\
\hline $\begin{array}{l}\text { Develop Indirect Pool FY } 1996 \\
\text { Execution Rates }\end{array}$ & $\begin{array}{l}\text { Coordinate and develop a submittal detailing the indirect FY } 1996 \\
\text { execution rates. }\end{array}$ \\
\hline $\begin{array}{l}\text { Fy } 1995 \text { Indirect Monthly Summaries } \\
\text { and Periodic Reconciliation of } \\
\text { Targets }\end{array}$ & $\begin{array}{l}\text { Provide RL with approved, final copies of the FY } 1995 \text { SSPP WBS } \\
\text { Dictionaries. }\end{array}$ \\
\hline $\begin{array}{l}\text { WHC Indirect Pool Accumulation } \\
\text { Distribution Activity Handbook }\end{array}$ & $\begin{array}{l}\text { Update and revise the WHC indirect pool accumulation/distribution } \\
\text { activity (pool) for RL approval. This summary will be maintained } \\
\text { on Soft Reporting under "FDS Guideline Handbook" in Section } 4 \text {, } \\
\text { "Internal Services (Pools) Descriptions". }\end{array}$ \\
\hline $\begin{array}{l}\text { Submit Monthly Company-Level } \\
\text { Overhead SMS Report (Executive } \\
\text { Indirect, Expense, Capital, and } \\
\text { Line Item Summaries and } 6.10 .12 \\
\text { Program Data) }\end{array}$ & $\begin{array}{l}\text { Submit monthly an SMS report which includes a Company-level } \\
\text { Overhead Executive Indirect, Capital, Line Items, and Expense } \\
\text { Summaries and } 6.10 .12 \text { program data. }\end{array}$ \\
\hline Indirect Al10cable Cost Report & Coordinate and develop a submittal report to $\mathrm{RL}$. \\
\hline $\begin{array}{l}\text { Add WHC Financial Curriculum } \\
\text { courses to the Training Resource } \\
\text { and Data Exchange (TRADE) } \\
\text { electronic course catalog }\end{array}$ & $\begin{array}{l}\text { Implementation and coordination of a course schedule in the TRADE } \\
\text { electronic course catalog. This electronic BBS displays the course } \\
\text { listings to all DOE and its contractors. }\end{array}$ \\
\hline $\begin{array}{l}\text { Develop Outyear Company Level } \\
\text { Overhead Planning Rates }\end{array}$ & $\begin{array}{l}\text { Coordinate and develop a submittal detailing the indirect FY } 1996 \\
\text { through FY } 2001 \text { ADS planning rates. }\end{array}$ \\
\hline
\end{tabular}




\begin{tabular}{|c|c|c|c|c|}
\hline \multicolumn{3}{|c|}{ 2.B.1 Milestone List } & $\begin{array}{c}\text { Westinghouse Hanford Company } \\
\text { Finance and Administration } \\
\text { SMS/WBS } 6.10\end{array}$ & $\begin{array}{c}\text { FY } 1995 \\
\text { Site Support Program Plan }\end{array}$ \\
\hline \multicolumn{2}{|c|}{ Milestone } & \multirow{2}{*}{$\begin{array}{l}\text { WBS } \\
\text { Number }\end{array}$} & \multirow[b]{2}{*}{ Milestone Description } & \multirow{2}{*}{$\begin{array}{l}\text { Due } \\
\text { Date }\end{array}$} \\
\hline Type & Number & & & \\
\hline DOE-RL & $\begin{array}{l}\text { FAB-95-001 } \\
\text { thru } 012\end{array}$ & 6.10 .1 & $\begin{array}{l}\text { Monthiy } 6.10 .1 \text { Site } \\
\text { Management System } \\
\text { report. }\end{array}$ & $\begin{array}{l}3 \text { rd } \\
\text { Fiscal } \\
\text { Friday } \\
\text { of Month }\end{array}$ \\
\hline DOE-RL & FAB-95-013 & 6.10 .1 & $\begin{array}{l}\text { FY } 1996 \text { Site Support } \\
\text { Program Plan }\end{array}$ & $08 / 31 / 95$ \\
\hline DOE-RL & FAB-95-014 & 6.10 .1 .1 & Unified Budget Call & TBD \\
\hline DOE-RL & $\begin{array}{l}\text { FAB-95-015 } \\
-016 \\
\end{array}$ & 6.10 .1 .1 & $\begin{array}{l}\text { Budget Manual draft \& } \\
\text { final report }\end{array}$ & $\begin{array}{l}10 / 14 / 94 \\
12 / 02 / 94 \\
\end{array}$ \\
\hline DOE-RL & $\begin{array}{l}\text { FAB-95-017 } \\
\text { thru } 019\end{array}$ & 6.10 .1 .1 & $\begin{array}{l}\text { Systematic Uncosted } \\
\text { obligations Analyses }\end{array}$ & $\begin{array}{l}11 / 30 / 94 \\
05 / 30 / 95 \\
09 / 29 / 95 \\
\end{array}$ \\
\hline DOE-RL & $\begin{array}{l}\text { FAB-95-020 } \\
\text { thru } 024\end{array}$ & 6.10 .1 .1 & $\begin{array}{l}\text { Uncosted Variance } \\
\text { Analysis }\end{array}$ & $\begin{array}{l}05 / 19 / 95 \\
06 / 16 / 95 \\
07 / 14 / 95 \\
08 / 18 / 95 \\
09 / 15 / 95\end{array}$ \\
\hline DOE-RL & $\begin{array}{l}\text { FAB-95-025 } \\
\text { thru } 027\end{array}$ & 6.10 .1 .1 & $\begin{array}{l}\text { Outlay Estimate Draft } \\
\text { Revisions, FY95 Final, } \\
\text { Estimate for FY96 and } \\
\text { Quarterly Outlay } \\
\text { Variance Reports (as } \\
\text { needed) }\end{array}$ & $\begin{array}{l}12 / 06 / 94 \\
03 / 07 / 95 \\
06 / 06 / 95\end{array}$ \\
\hline
\end{tabular}




\begin{tabular}{|c|c|c|c|c|c|}
\hline \multicolumn{3}{|c|}{ 2.B.1 Milestone List } & \multicolumn{2}{|c|}{$\begin{array}{c}\text { Hestinghouse Hanford Company } \\
\text { Finance and Administration } \\
\text { SMS/WBS } 6.10\end{array}$} & $\begin{array}{c}\text { FY } 1995 \\
\text { Site Support Program Plan }\end{array}$ \\
\hline \multicolumn{2}{|c|}{ Milestone } & \multirow[b]{2}{*}{$\begin{array}{l}\text { WBS } \\
\text { Number }\end{array}$} & \multirow{2}{*}{\multicolumn{2}{|c|}{ Milestone Description }} & \multirow[b]{2}{*}{$\begin{array}{l}\text { Due } \\
\text { Date } \\
\end{array}$} \\
\hline Type & Number & & & & \\
\hline DOE-RL & $\begin{array}{l}\text { FAB-95-028 } \\
\text { thru } 031\end{array}$ & 6.10 .1 .1 & $\begin{array}{l}\text { Internal Evaluation/ } \\
\text { Surveill ance Schedule, } \\
\text { Reports, and Corrective } \\
\text { Action Plans }\end{array}$ & & $\begin{array}{l}10 / 14 / 94 \\
01 / 16 / 95 \\
04 / 14 / 95 \\
07 / 14 / 95 \\
\end{array}$ \\
\hline DOE-RL & $\begin{array}{l}\text { FAB-95-032 } \\
-033 \\
\end{array}$ & 6.10 .1 .1 & FIVRS Cost Estimate & & $\begin{array}{l}10 / 14 / 94 \\
04 / 14 / 95 \\
\end{array}$ \\
\hline$D O E-R L$ & $\begin{array}{l}\text { FAB-95-034 } \\
\text { thru } 037\end{array}$ & 6.10 .1 .1 & $\begin{array}{l}\text { Transfers To/From Other } \\
\text { Hanford Contractors : }\end{array}$ & & $\begin{array}{l}10 / 14 / 94 \\
01 / 16 / 95 \\
04 / 14 / 95 \\
07 / 14 / 95 \\
\end{array}$ \\
\hline DOE-RL & $\begin{array}{c}\text { FAB-95-038 } \\
\text { thru } 049 \\
\end{array}$ & 6.10 .1 .1 & $\begin{array}{l}\text { Monthly Budget CFO } \\
\text { Briefing Report }\end{array}$ & & TBD \\
\hline $\mathrm{DOE}-\mathrm{RL}$ & $\begin{array}{l}\text { FAB-95-050 } \\
\text { thru } 053\end{array}$ & 6.10 .1 .2 & $\begin{array}{l}\text { SAS Obligation and Cost } \\
\text { Report }\end{array}$ & & $\begin{array}{l}10 / 14 / 94 \\
01 / 16 / 95 \\
04 / 14 / 95 \\
07 / 14 / 95 \\
\end{array}$ \\
\hline DOE-RL & FAB-95-054 & 6.10 .1 .3 & $\begin{array}{l}\text { Overhead Planning Self } \\
\text { Surveillance for } \\
\text { Improvements to the FY } \\
1995 \text { Indirect Planning } \\
\text { Process }\end{array}$ & & $07 / 31 / 95$ \\
\hline
\end{tabular}




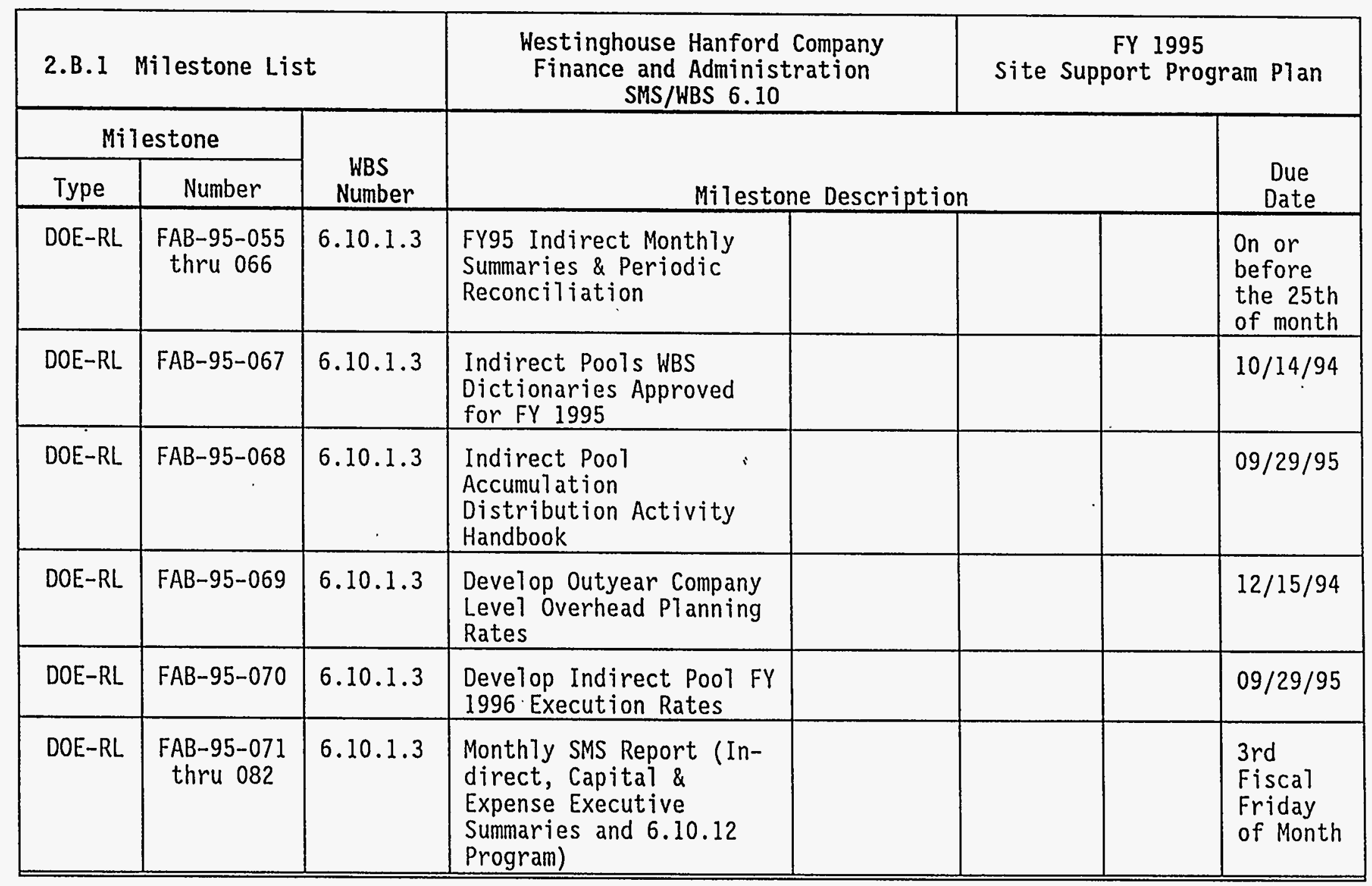




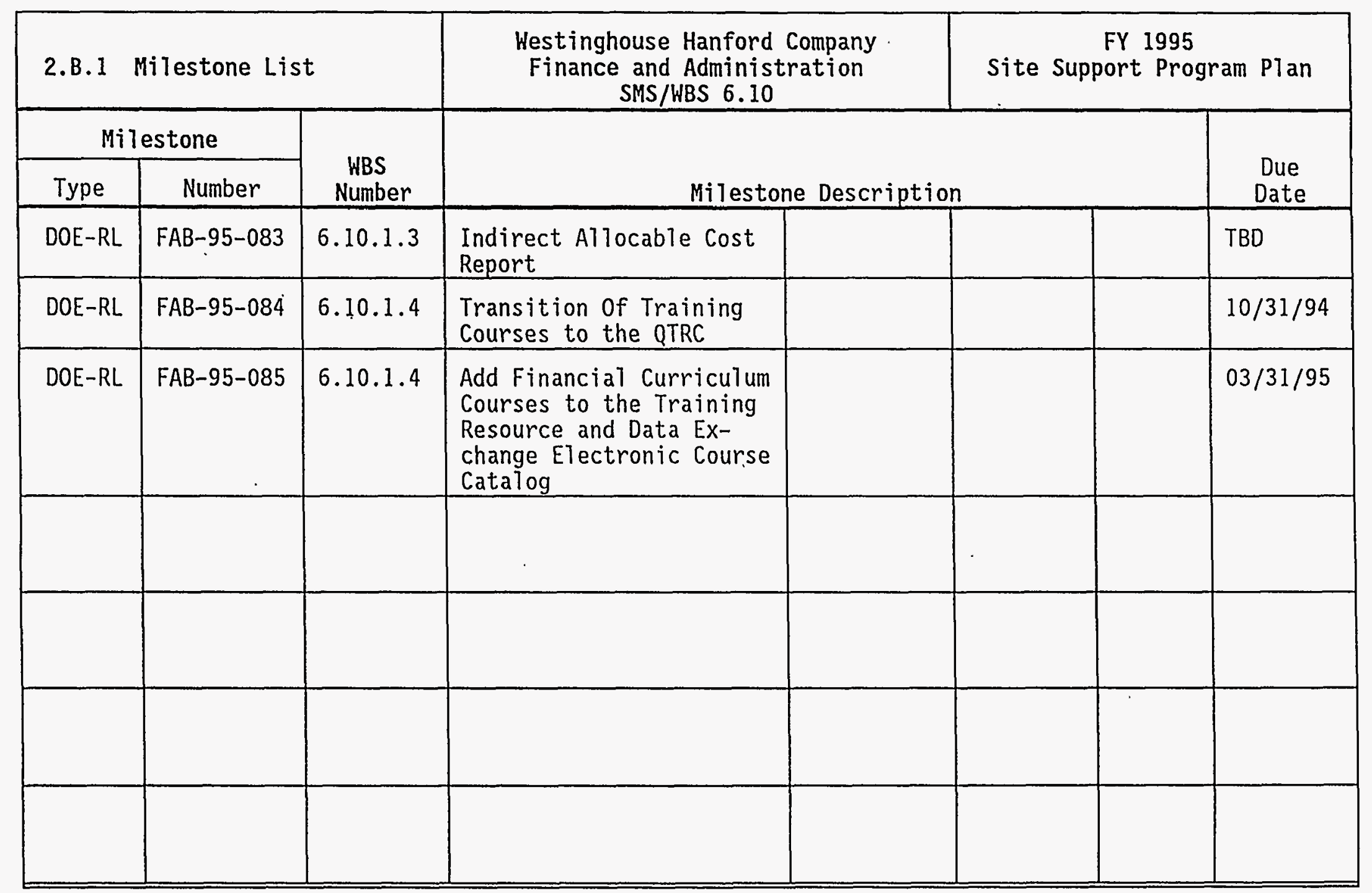


2.C.1.1 Cost Baseline by Program Element 2.C.1.2 Cost Baseline by Funding Source

Westinghouse Hanford Company Finance and Administration SMS/WBS 6.10
FY 1995 SSPP

FY 1994 COST BASELINE (Do 11 ars in Thousands)

\begin{tabular}{|c|c|c|c|c|c|c|c|}
\hline WBS \# & Title & $\mathrm{DOH}$ & CSP/SWS & SLP & $G \& A$ & Direct $\$$ & TOTAL \$S \\
\hline 6.10 .1 .1 & Financial Integration \& Planning & & & & $\$ 718.3$ & & $\$ 718.3$ \\
\hline 6.10 .1 .2 & Program Support SAS/Overhead Planning & & & & $\$ 746.7$ & & $\$ 746.7$ \\
\hline & & & & & & & \\
\hline & & & & & & & \\
\hline & TOTALS & & & & $\$ 1465.0$ & & $\$ 1465.0$ \\
\hline
\end{tabular}

FY 1995 COST BASELINE (Dollars in Thousands)

\begin{tabular}{|c|c|c|c|c|c|c|c|}
\hline WBS \# & Title & $\mathrm{DOH}$ & CSP/SWS & SLP & $G \& A$ & Direct \$ & TOTAL \$S \\
\hline 6.10 .1 .1 & Financial Integration \& Planning & & & & $\$ 476.0$ & & $\$ 476.0$ \\
\hline 6.10 .1 .2 & Financial Services & & & & $\$ 276.0$ & & $\$ 276.0$ \\
\hline 6.10 .1 .3 & Overhead Planning & & & & $\$ 699.0$ & & $\$ 699.0$ \\
\hline 6.10 .1 .4 & Financial Training & & & & $\$ 295.0$ & & $\$ 295.0$ \\
\hline & TOTALS & & & & $\$ 1746.0$ & & $\$ 1746.0$ \\
\hline
\end{tabular}


2.C.1.1 Cost Baseline by Program Element

Westinghouse Hanford Company

Finance and Administration

FY 1995

SMS/WBS 6.10

FY 1996 COST BASELINE (Dol Tars in Thousands)

\begin{tabular}{|c|c|c|c|c|c|c|c|}
\hline WBS \# & Title & $\mathrm{DOH}$ & CSP/SWS & SLP & $G \& A$ & Direct $\$$ & TOTAL \$S \\
\hline 6.10 .1 .1 & Financial Integration \& Planning & & & & $\$ 489.8$ & & $\$ 489.8$ \\
\hline 6.10 .1 .2 & Financial Services & & & & $\$ 284.0$ & & $\$ 284.0$ \\
\hline 6.10 .1 .3 & Overhead Planning & & & & $\$ 719.3$ & & $\$ 719.3$ \\
\hline 6.10 .1 .4 & Financial Training & & & & $\$ 303.5$ & & $\$ 303.5$ \\
\hline & TOTALS & & & & $\$ 1796.6$ & & $\$ 1796.6$ \\
\hline
\end{tabular}

FY 1997 COST BASELINE (Dollars in Thousands)

\begin{tabular}{|c|c|c|c|c|c|c|c|}
\hline WBS \# & TitTe & $\mathrm{DOH}$ & CSP/SWS & SLP & $G \& A$ & Direct $\$$ & TOTAL \$S \\
\hline 6.10 .1 .1 & Financial Integration \& Planning & & & & $\$ 519.2$ & & $\$ 519.2$ \\
\hline 6.10 .1 .2 & Financial Services & & & & $\$ 301.0$ & & $\$ 301.0$ \\
\hline 6.10 .1 .3 & Overhead Planning & & & & $\$ 762.5$ & & $\$ 762.5$ \\
\hline 6.10 .1 .4 & Financial Training & & & & $\$ 321.7$ & & $\$ 321.7$ \\
\hline & TOTALS & & & & $\$ 1904.4$ & & $\$ 1904.4$ \\
\hline
\end{tabular}




\begin{tabular}{|c|c|c|}
\hline 2.C.2 Cost Basis & $\begin{array}{c}\text { Westinghouse Hanford Company } \\
\text { Finance and Administration } \\
\text { SMS/WBS 6.10 }\end{array}$ & $\begin{array}{c}\text { FY } 1995 \\
\text { Site Support Program P1an }\end{array}$ \\
BUDGET \\
WBS 6.10.1
\end{tabular}

Labor cost based on the identified hours required to perform each analysts identifiable activities. Resulting FTE's are based on these hours and priced according to the labor rate guidance as reflected in the Financial Data System.

Non-labor cost elements, primarily service assessments, äre based on planning guidance from utilized services (i.e., BCS, etc..). Supplies, training and travel are based on consideration of historical usage plus quantitative knowns for the next fiscal year. 


\begin{tabular}{|c|c|c|c|c|c|c|c|c|}
\hline \multicolumn{2}{|c|}{ 2.C.3 Full Time Equivalents } & \multicolumn{4}{|c|}{$\begin{array}{c}\text { Westinghouse Hanford Company } \\
\text { Finance and Administration } \\
\text { SMS/WBS } 6.10 .1\end{array}$} & \multicolumn{3}{|c|}{$\begin{array}{c}\text { FY } 1995 \\
\text { Site Support Program PIan }\end{array}$} \\
\hline \multicolumn{4}{|c|}{ Ful1-Time Equivalent Staff by Job Description } & \multicolumn{5}{|c|}{ NOTE: Job Family Only.After 1996} \\
\hline JOB FAMILY & \%. & & अे। & 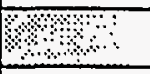 & & & & \\
\hline Job category & 1994 & 1995 & 1996 & 1997 & 1998 & 1999 & 2000 & 2001 \\
\hline MANAGERS $\quad \%$ & ל. & & \%: & +3 & 3 & 3 & 3 & 3 \\
\hline First line & 2 & 2 & 2 & & & & & \\
\hline General/executive & & 1 & 1 & & & & & \\
\hline \multicolumn{9}{|l|}{ Project/Program } \\
\hline ADMIN/OTHER RROEESSTONALS & 1. & & \%) & 2.18 .9 & 18.9 & 18.9 & 18.9 & 18.9 \\
\hline Accountant/auditor & 15 & 18.9 & 18.9 & & & & & \\
\hline \multicolumn{9}{|l|}{ Computer System Anal . } \\
\hline \multicolumn{9}{|l|}{ Cost Est/planner/sch } \\
\hline GEN ADM/SECRETARY ILERK $/$ । & 18. & & ?.। & 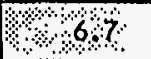 & 6.7 & 6.7 & 6.7 & 6.7 \\
\hline \multicolumn{9}{|l|}{ Admin Assistants } \\
\hline office Clerks (Gen) & 2 & 3.4 & 3.4 & & & & & \\
\hline Office Clerks (Special) & 1 & 1 & 1 & & & & & \\
\hline Secretaries & & 1 & 1 & & & & & \\
\hline \multicolumn{9}{|l|}{ Typist/Hord Process } \\
\hline other & & 1.3 & 1.3 & & & & & \\
\hline
\end{tabular}




\begin{tabular}{|c|c|c|}
\hline $\begin{array}{l}\text { wate Management } \\
\text { System }\end{array}$ & WESTINGHOUSE HANFORD COMPANY \\
6.10 .1 BUDGET & 1995 \\
\hline
\end{tabular}

MILESTONE SCHEDULE

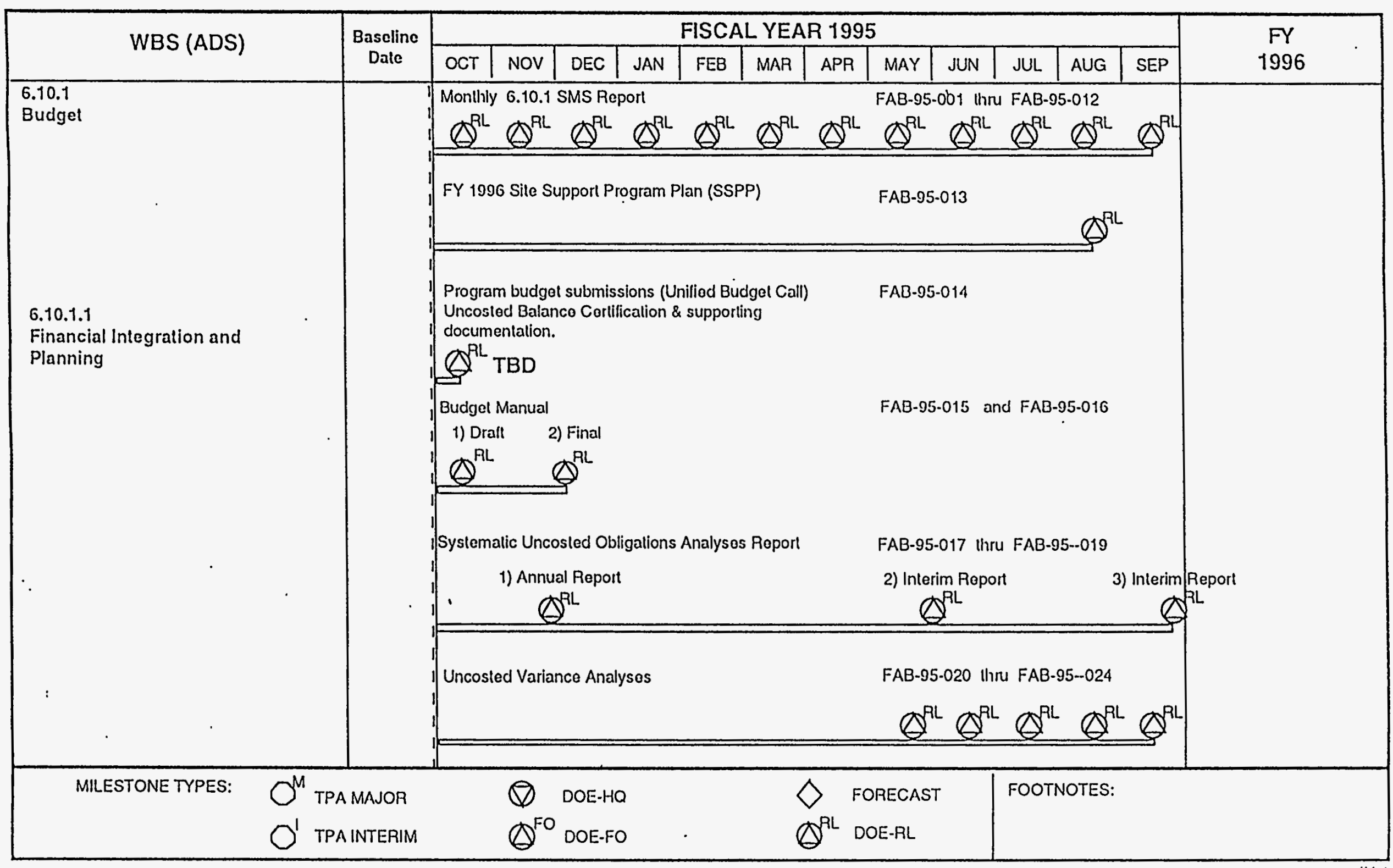




\begin{tabular}{|c|c|c|c|}
\hline & $\begin{array}{l}\text { anagement } \\
\text { System }\end{array}$ & $\begin{array}{c}\text { WESTINGHOUSE HA JRD COMPANY } \\
6.10 .1 \text { BUDGET }\end{array}$ & 1995 \\
\hline
\end{tabular}

\section{MILESTONE SCHEDULE}

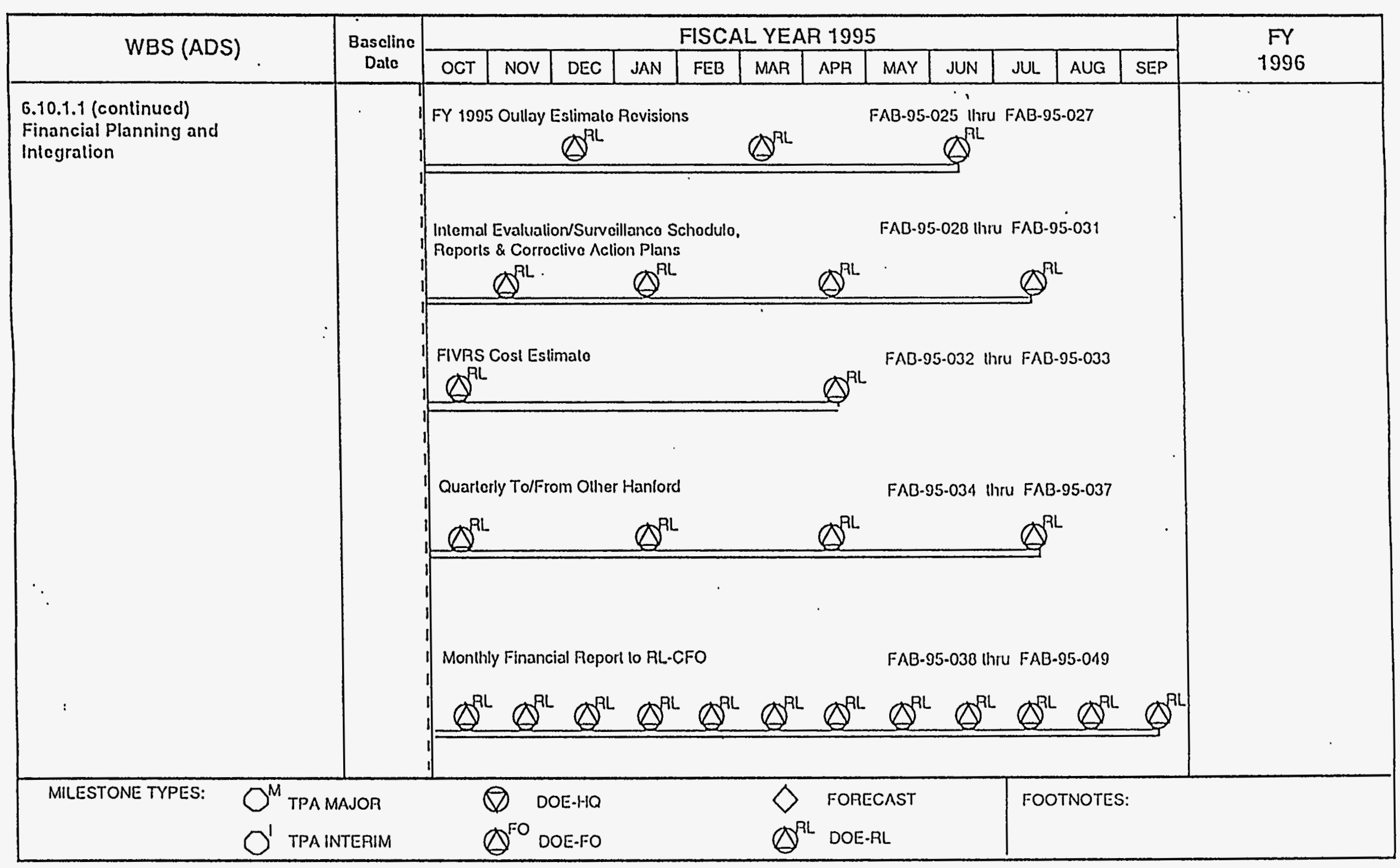




\begin{tabular}{|c|c|c|c|c|}
\hline is & $\begin{array}{l}\text { inagement } \\
\text { system }\end{array}$ & 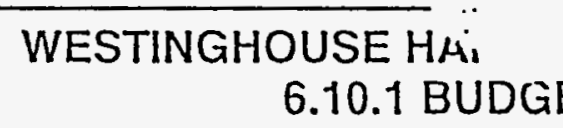 & $\begin{array}{l}\text { RD COMPANY } \\
\text { ET }\end{array}$ & 1995 \\
\hline
\end{tabular}

\section{MILESTONE SCHEDULE}

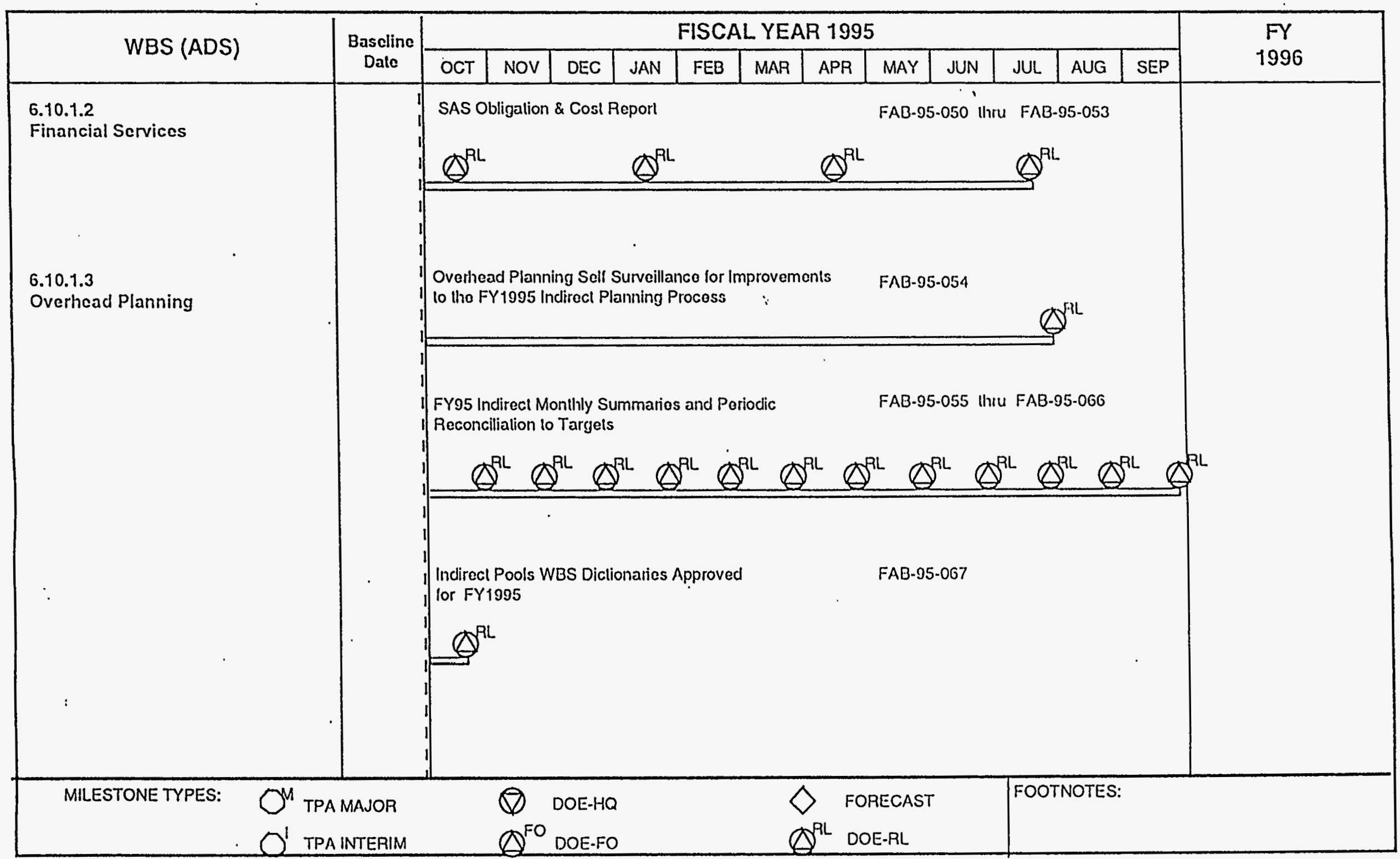




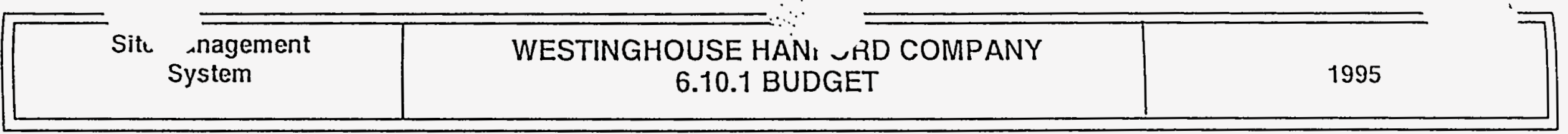

\section{MILESTONE SCHEDULE}

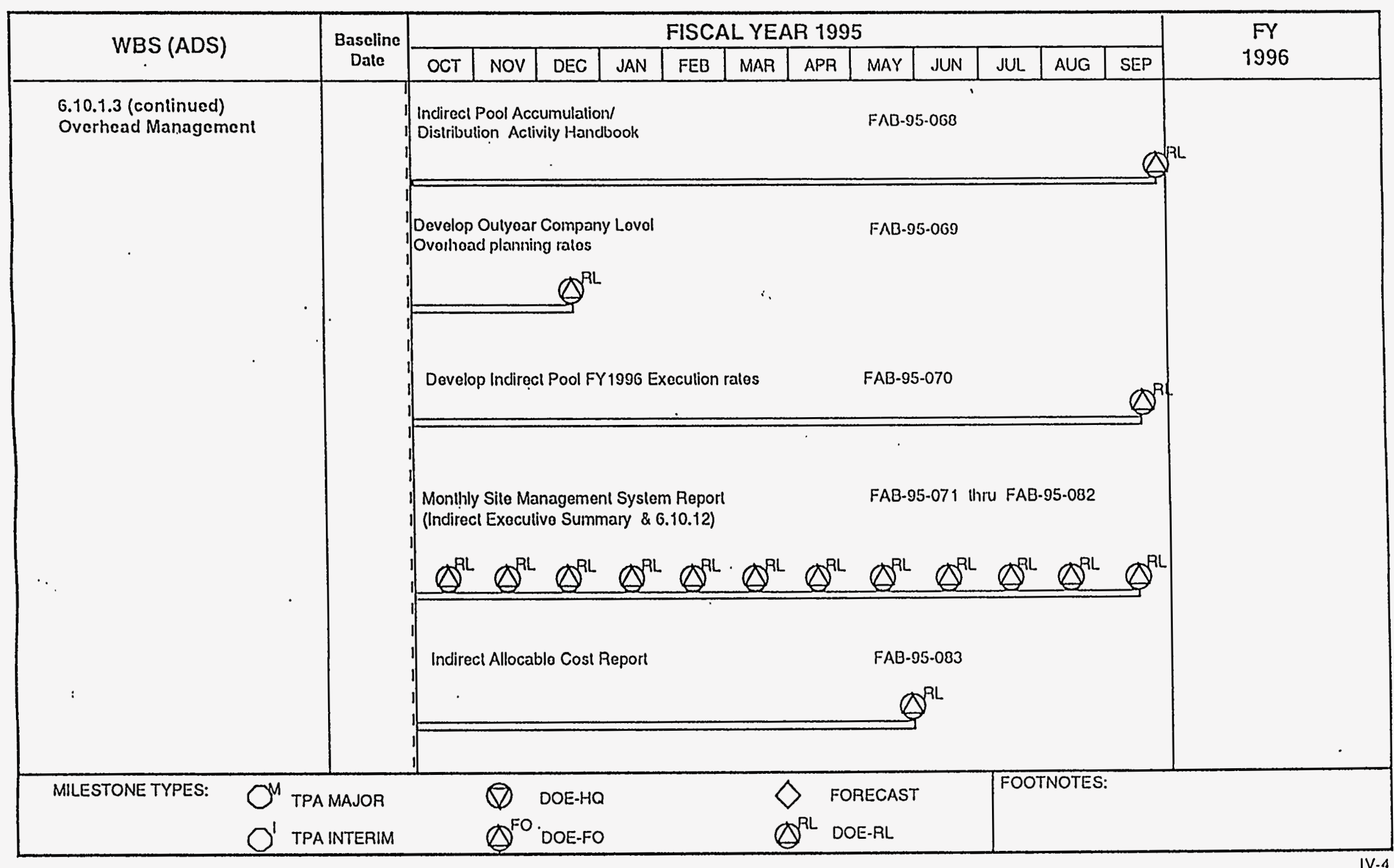




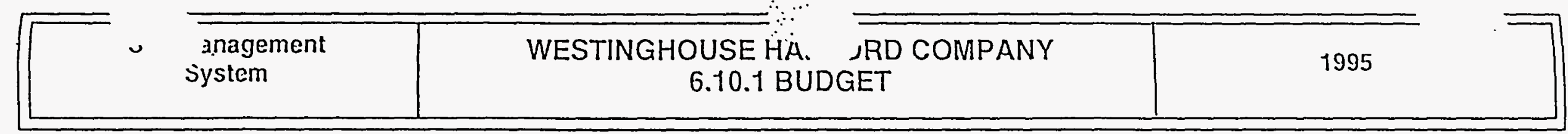

\section{MILESTONE SCHEDULE}

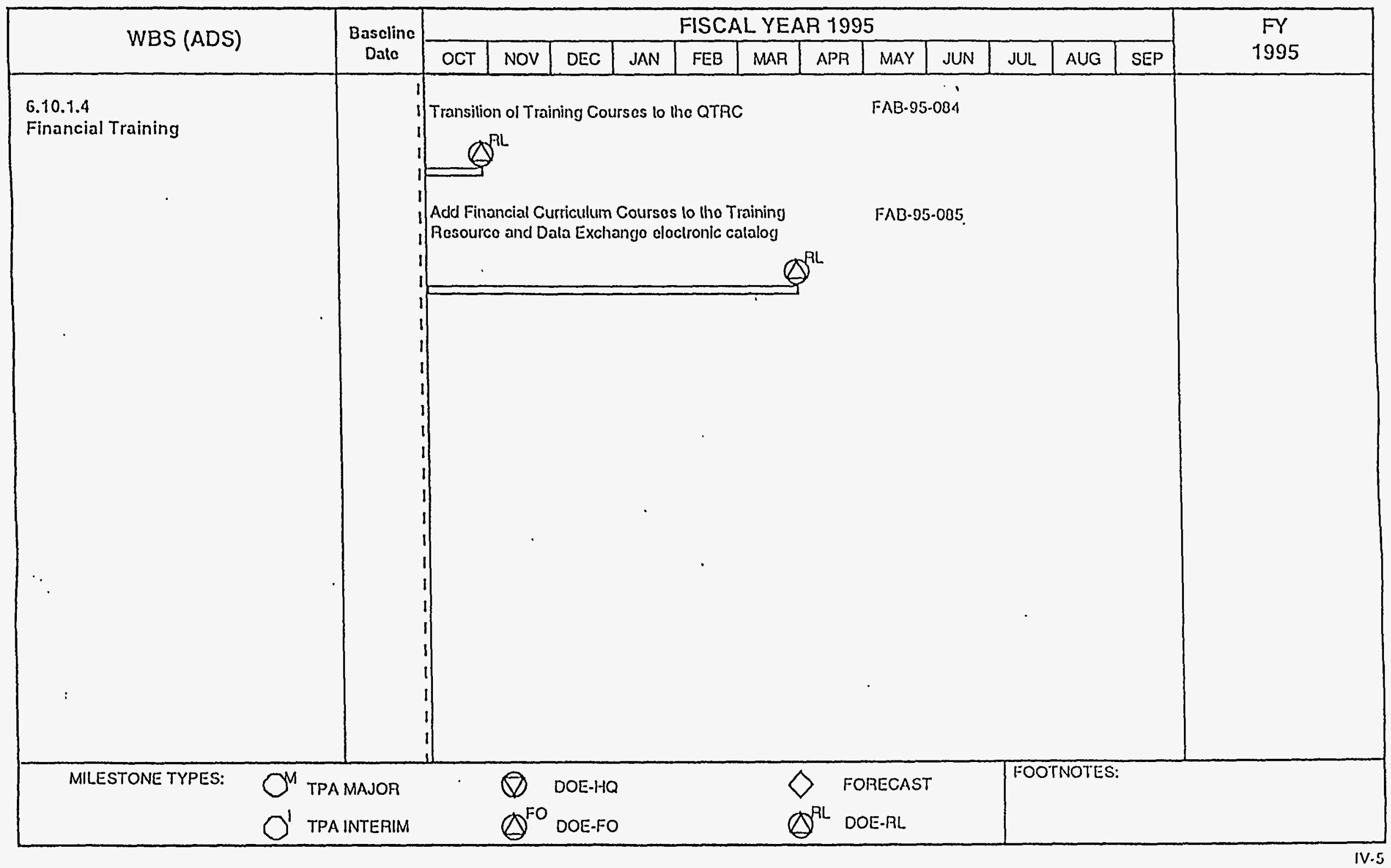




\section{WORK STATEMENT -}

REQUESTS WITHIN TARGET:

Activity Detailed Description

Financial Services is staffed with one manager. Responsibilities include line management and leadership to assure the mission and objectives of the Controller's organization are accomplished.

Financial Services is staffed with one part-time summer intern to provide general office support. Responsibilities include mail distribution, scheduling, report/spreadsheet preparation, and general assislance to staff.

Financial Services is staffed with three exempt Project Control Analysts to provide oversight and coordination of designated WHC budgetary and manpower planning aclivities directed by the Controller's organization. Included are cost account development, preparation, and maintenance; cost and commitment controls; program analysis; and overall financial support. These analysts were funded in 1 MDO 15 in FY94. The 4.7 FTEs in FY94 were SAS FTEs supporting budget functions.

Financial Services also supports other organizations within WHC. ESQ Services organization support includes one manager and seven project control analysts which provide overall financial support to the Emergency Preparedness, Safegaurds and Security, Fire Department, ESQ Program Office, Safety, Health Physics, Compliance Assurance, and Quality Assurance groups. P\&MM support includes one analyst for overall financial support. Economic Transition organization support includes one analyst for overall financial support.

The decrease in labor cost from FY94 to FY95 is attributed to a re-alignment of staff. The SAS financial analysts will be funded direclly by Safegaurds and Security. Financial Services analysts and management funded in 1MDD 15 Financial Services in FY94 are now funded in 1 MDD 16 Budgets, with the exception of analysts funded by ESQ.

\begin{tabular}{|c|c|c|c|c|c|c|c|}
\hline \multicolumn{2}{|c|}{ FY 1994 FYSF } & \multicolumn{2}{|c|}{ Delta } & \multicolumn{2}{|c|}{ FY 1995} & \multicolumn{2}{|c|}{ Delta } \\
\hline FTES & Dollars & FTES & Dollars & FTEs & Dollars & FTES & $\mathrm{D}$ \\
\hline
\end{tabular}

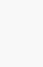

s

Total FTE/Labor Cost Total Non-Labor Cost 


\begin{tabular}{|l|}
\hline $\begin{array}{l}\text { COS } \\
\text { IMD }\end{array}$ \\
\hline $\begin{array}{l}\text { Work Breakdown } \\
\text { Structure } \\
\text { Dictionary }\end{array}$ \\
\hline
\end{tabular}

\section{GOST CONTENT}

Overhead Planning is funded in the G\&A Pool. Major costs are management; analytical and clerical support; office supplies; purchased services such as training and travel; site services such as QTRC training; and BCS maintenance support.

\section{TECHNICAL CONTENT}

Overhead Planning provides overall coordination and baseline control of indirect budget/liquidation rate planning for WHC, in accordance with cost accounting standards (CAS), generally accepted accounting principles (GAAP), the WHC Disclosure Statement, and WHC/RL reporting requirements. Overhead Planning provides financial interface with other Hanford Contractors and supports budget validalion efforts by DOE-RL Laboratory and Overhead Branch.

\section{OBJECTIVES - (Specific)}

- Maintaln company-level tracking of all current year indirect funding, fiscal year spending forecasts, liquldations, and rates and variance management.

- Assertively manage indirect activities in the most cost effective manner.

- Provide financial planning processes and oversee the development of indirect budgets to ensure they support programmatic missions and requirements.

- Monitor and manage the authorized spending levels of company-level overhead to ensure RL issued cellings are not exceeded.

- Evaluate the effectiveness of the internal indirect budget development process.

- Coordinate review/analysis of direct versus indirect funding determinations.

- Monitor indirect change requests and provide assessment of impact to RL company-level overhead funding targets, rates, and ECCEL.

- Coordinate indirect pools budget requests for approval.

- Submit indirect liquidation rates to RL-FMD for approval.

- Request changes in company-level overhead and service pool liquidation methodologies, if appropriate.

\section{OBJECTIVES - (General)}

- Conduct all operations in a safe and cost-effective manner.

- Meet RL-Laboratory and Overhead Branch requirements and commitments.

- Manage all activities in a total quality manner.

- Develop and maintain policles and procedures to ensure effective Internal controls for all indirect funds.

- Provide oversight/coordination of all WHC indirect budgetary activities.

- Develop and maintain company-level indirect profiles; and prepare responses involving budgetary data as requested by RL, Management, DOE-HQ, $\mathrm{OMB}$, Congress, the Medla, and/or the public. 


\begin{tabular}{l} 
COS: \\
IMDD16 \\
\hline
\end{tabular}

Work Breakdown

Structure

Dictionary

Westinghouse Hanford Company

6.10.1 BUDGET

Part II - Element Definition
FY 1995 SSPP

Revision \# 0

\begin{tabular}{|lllll}
\hline WBS ELEMENT CODE: & 6.10 .1 .3 & TITLE: & Overhead Planning \\
\hline
\end{tabular}

\section{ELEMENT TASKDESCRIPTION}

\section{OBJECTIVES - (General continued)}

- Develop and maintain procedures and funding controls to ensure effective indirect startup and closeout of each fiscal year.

- Ensure that all staff responsible for preparing or reviewing indirect budgets are trained to adhere to proper financial and budgetary standards.

- Perform activities to ensure effective utilization and proper control/execution of all indirect funds.

- Monitor fiscal year spending and liquidation forecasts.

- Provide adequate oversight through surveillances and reviews, and internal audits based on an analysis of audit risks.

\section{ASSUMPTIONS/CONSTRAINTS -}

- Indirect budget funding baselines should be consistent with FY funding levels as Identified in guidance received from RL-Laboratory and Overhead Branch.

- Activities must comply with current applicable regulations and guldance: Federal/State Regulations; DOE Contract; DOE Orders and Directives; DOE/RL Guidance/Correspondence; Labor Contracts; WHC Management Guidance; Management Requirements and Procedures (MRP), Management Policies and other Controlled Manuals.

\section{MILESTONES -}

- Coordinate and submit Indirect Allocable Cost Report

- Reconcllation of WBS Diclionaries to Indirect Funding Targets for FY 1995

- Submit Indirect Pools WBS Dictionaries approved for FY 1995

- WHC Indirect Pool Accumulation/Distribution Activity (Pool) Handbook (A section in the Budget Guideline Handbook)

- Company-Level Overhead Planning Rates for FY 1996 thru 2001

- Develop all Indirect Pool FY 1996 Executions Rates

- Overhead Planning Self-Surveillance for improvements to the FY 1995 indirect planning process

\section{: DELIVERABLES -}

- Monthly Site Management System indirect Executive Summary Report

- Allocable Cost Report

- Periodic SMS 6.10.12 Reports 


\section{WORK STATEMENT -}

\section{REQUESTS WITHIN TARGET:}

\section{Activity Detailed Description}

Overhead Planning is staffed with one manager. Responsibilities include staffing, management, Call Lelter planning, CFO briefings, recruitment coordination, and leadership to assure the mission and objectives are accomplished within the scheduled milestones and within budget constraints.

Overhead Planning is staffed with one full-time clerical and one part- time COE student to provide general office administration and accountability. Other duties include maintaining/iling budget documentation, desk procedures, training development, change log update, safely meetings, typing and associated clerical functions.

Overhead Planning is staffed with 3 exempt Project Control Analysts for oversight and coordination of all WHC indirect budgetary activities.

One analyst provides complete coordination and analysis of service pool assessments and distributions. Other activities include BCS contractor interface, rates/assessment documentation, recruiting support, coordination of business rotational and summer intern assignments, monthly SMS reporting, mid-year review and analysis of assigned cost accounts.

One analyst provides complete coordination and analysis of the department overhead assessments. Other aclivities include ICF-KH contractor interface, allocable cost reporting, coordination of the monthly indirect budget RL summary, mid-year review, analysis of assigned cost accounts, and quarterly analysis of Continuity of Service.

One analyst provides coordination and analysis of the company level overhead assessment and liquidations. Other activities include monthly rate analysis of rates, preparation of SMS reports, development of out year planning rates, indirect ECCEL resene coordination, PNL contractor interface, monthly SMS reporting, mid-year review and analysis of cost accounts.

-




$-$

\section{WORK STATEMENT -}

\section{REQUESTS WITHIN TARGET:}

\section{Activity Detailed Description (continued)}

Overhead Planning is staffed with 6 exempt Project Control Analysts within the Controller Business rotational program. Each of these analysts are assigned three-month rotational positions within the financial organizations. Each position is unique to the manager or department in which each reports. Common rotational positions include General Accounting, Internal Audit, Boeing Computer Services and waste management programs as required. Workscope includes budget planning and analysis, training and procedure documentation and special iequests as required.

Fiscal Year 1995 will include the Business Rotational employees in the $1 M D D 16$ cost account. In prior years, the rotationals were included in 1MDD 15, Financial Services. A corresponding decrease in labor costs will be applicable to 1MDD 15 in FY95. Additionally, one analyst transferred in FY94 and will not be replaced in FY95.

Costs are expected to remain level into the out-years with no significant change in the projected labor and non-labor elements. Furniture procurements that occurred in FY94 will not occur in FY95, however, addition of rotational analysts and their associated non-labor costs attribute to the increase in these cost elements.

Total FTE/Labor Cost Total Non-Labor Cost Total Overhead Planning $\%$

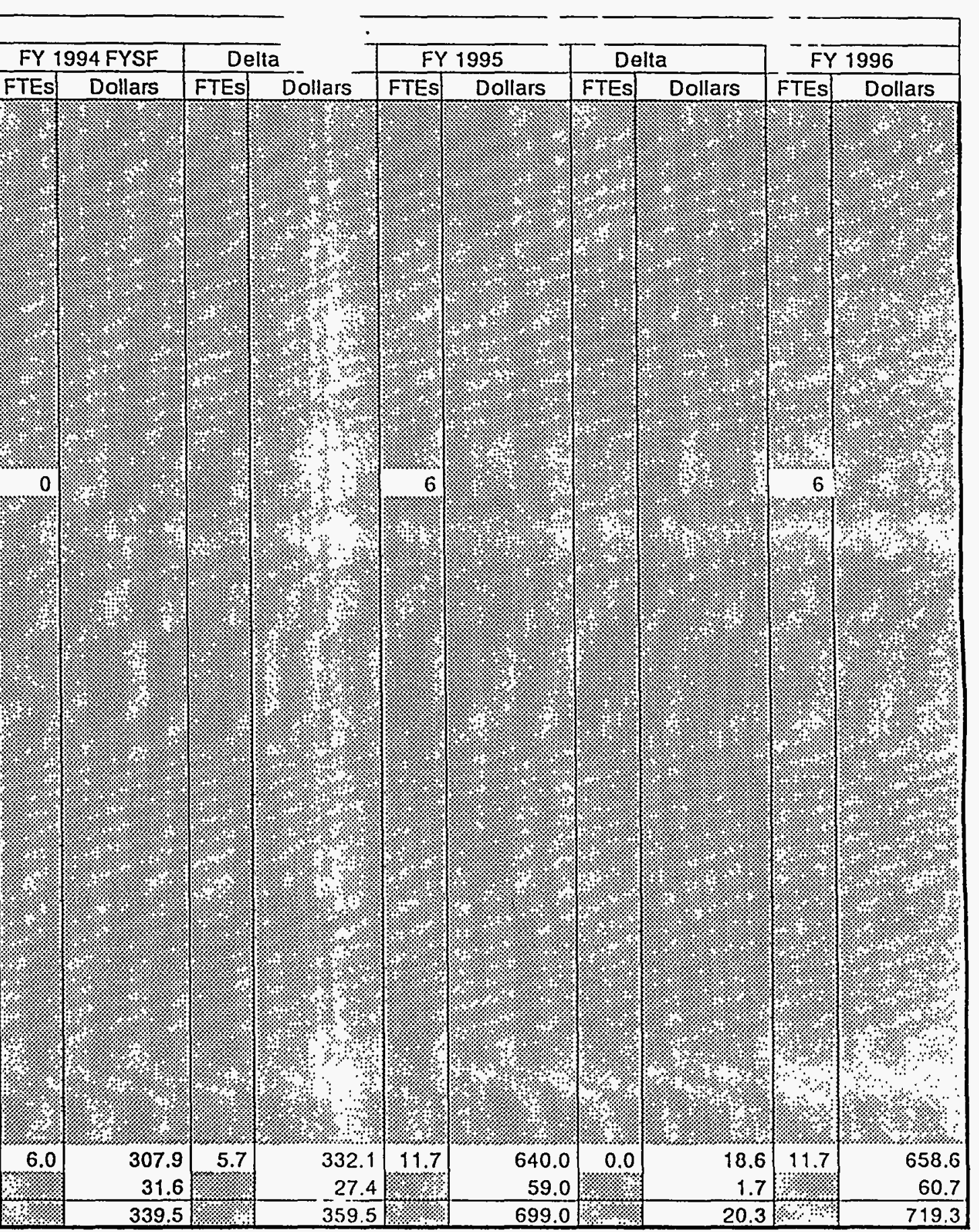




\section{$=\frac{1 M D_{1}}{\text { Work Breakdown }}$ Structure Dictionary}

\section{Westinghouse Hanford Company 6.10.1 BUDGET Part II - Element Definition}

FY 1995 SSPP

Revision \# 0

\section{WBS ELEMENT CODE: $\quad 6.10 .1 .4$}

ELEMENT TASKDESCRIPTION

\section{COST CONTENT -}

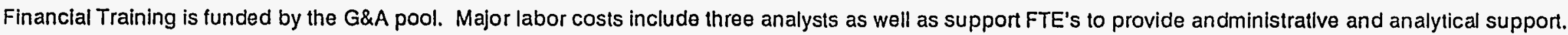

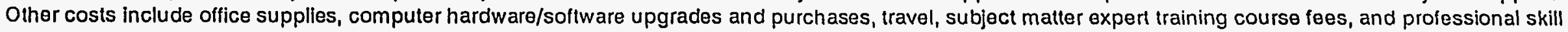
development training for the staff.

\section{TECHNICAL CONTENT -}

A three-way partnership was arranged to develop the Financial Curriculum training courses. This WBS describes the Line Managment Ownership (content owners) who have the lead role in providing coordination, facilitation, and clerical support to volunteer subject matter experts. The other partners, WHC's Leadership and Organization Development group provides change management and consulting expertise and the other partner, the Quality Training and Resource Center (QTRC) provides instructional design expertise, material productlon, and logistical support (registration, schoduling, elc.).

\section{OBJECTIVES}

- The primary objective is to complete the learning experiences currently in development to be offered to all Hanford site contractors, Including DOE's Richland Operations Office.

- Upon meeting the first objective, Financlal Training will share processes and products with other WEC GOCO's (Savings Through Sharing), as well as with the other contractors and DOE Field Offices within the DOE complex.

- Conduct all operations in a safe and cost-efficient manner.

- Manage all activities in a total quality manner.

- Add listing of Financial Curriculum courses to the QTRC catalog and the Headquarters system called Department Training Information System (DTIS).

- Meet with RL's Budget Division Training coordinator once monthly for interface, exchange, and sharing.

\section{ASSUMPTIONS}

Direction of the training development will remain In line with the conclusions drawn from the initial Needs Assessment survey. The Needs Assessment justified the tralning need, as well as identified the specific financial management topical areas to be addressed by the Financial Curriculum.

\section{: MILESTONES}

- Complete Implementation of Partnershlp with the QTRC and upon completion of course development, turn courses over to the QTRC.

- Add Financial Curriculum courses to the Training Resource and Data Exchange Electronic (TRADE) bulletin board system electronlc catalog called Training Resource Catalog (TRC).

\section{DELIVERABLES}

- Completion of the Financial Curriculum learning experiences currently In development. 
REQUESTS WITHIN TARGET:

Activity Detailed Description

Financial Training is staffed with two exempt analysts for FY 1995. Responsibilities include coordination and facilitation to each course content design leam, reporting/budgeting, subject matter point of contact, in-house marketing, maintenance of catalog listings.

maintaining evaluation feedback system/processes, course conten improvement, defining technical/instruction criteria with instruclors, and coordination with the QTRC.

Financial Training is staffed with one full-time clerical staff to provide support to content design team by scheduling/preparing meetings, record documentation, agenda creation, action tracking, inventory and document organization, and general staff assistance such as mail distribution, typing, and copying.

Financial Training is part-time staffed by a graduate rotational analyst for a six month rotation that will provide facilitation and coordination support for the content design teams, and to assist in course development.

FTE's FTE's

Financial Training is part-time staffed by exempt QTRC personnel to provide instructional design and technical editing support. Non-exempt QTRC personnel will provide desktop publishing and registration/scheduling support.

Financial Training was funded in 1 MDD 15, Financial Services work plan in FY94. Realignment of labor per RL direction brings : these FTEs into 1MDD 16 for FY95. The increase of labor and non-labor costs to 1 MDD 16 is attributed to this realignment. A corresponding decrease in costs in 1 MDD 15 reflects overall costs within target.

FTE's

Total FTE/Labor Cost

\section{se}

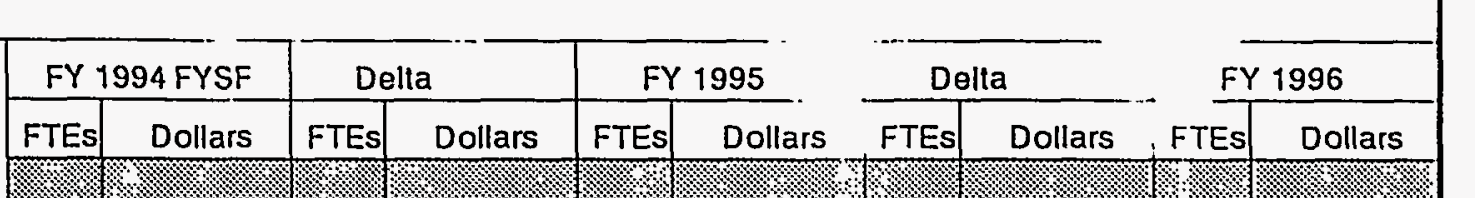

$\$$

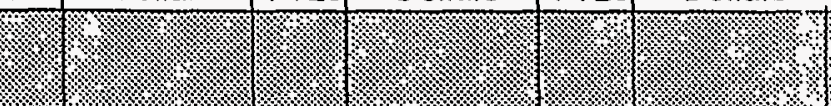

ฯ.

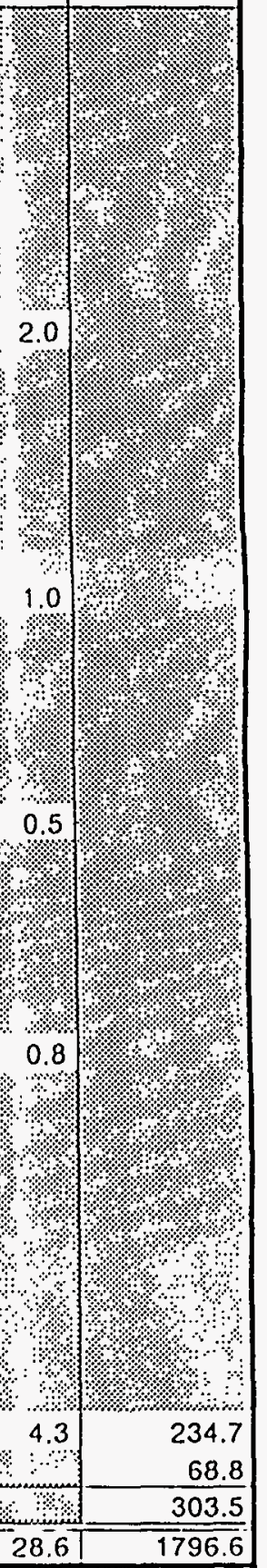




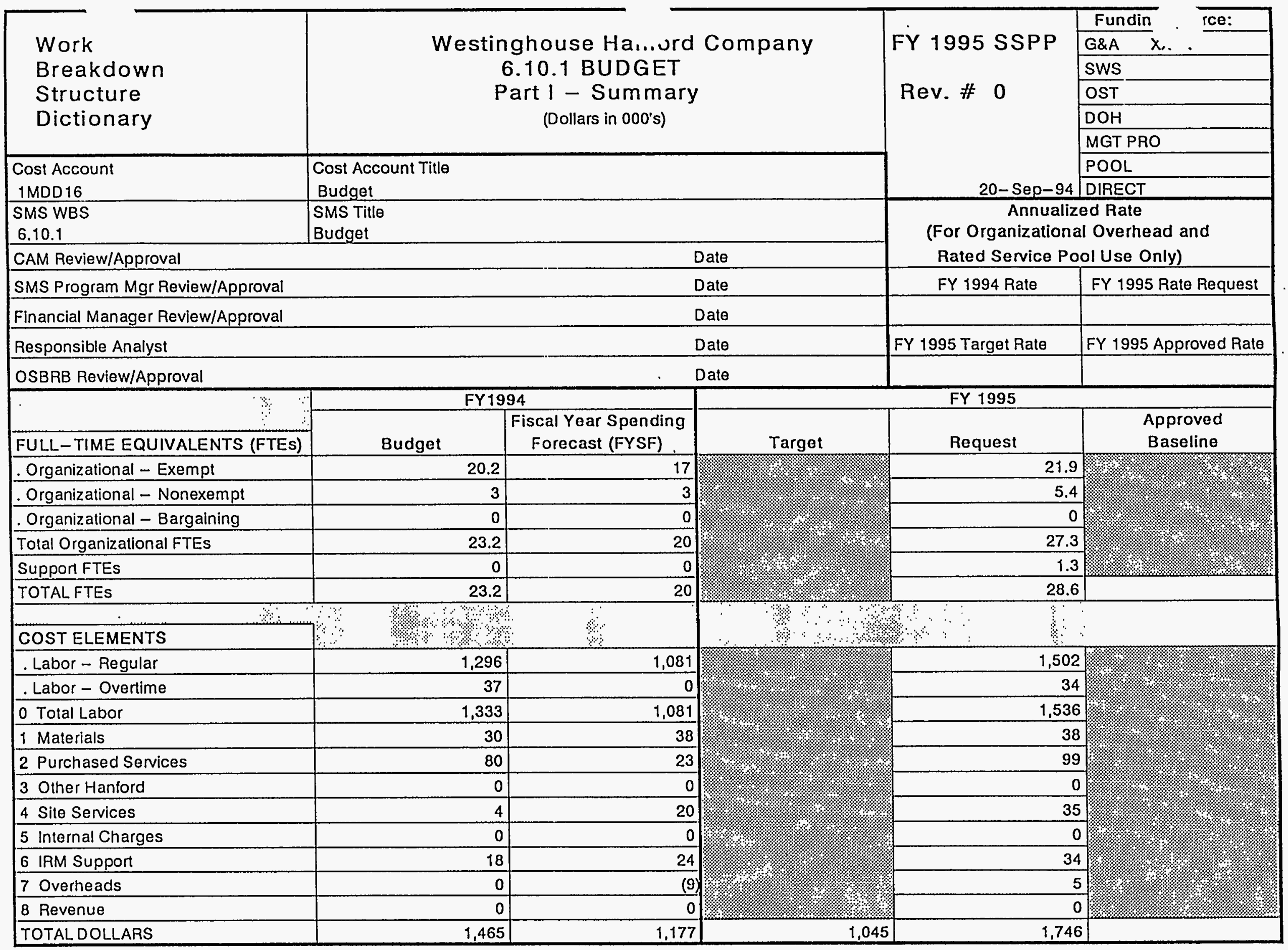




\begin{tabular}{l} 
COST UUNT \\
\hline \hline Work16 Breakdown \\
Structure \\
Dictionary \\
\hline
\end{tabular}

WBS ELEMENT COODE: 6.10 .1 .1
$\begin{aligned} & \text { ELEMENT TASK DESCRIPTION } \\ & \text { COST CONTENT }\end{aligned}$

\section{Westinghouse Hanford Company \\ 6.10.1 BUDGET}

Part II - Element Definition

FY 1995 SSPP

Revision \# 0

Financial Integration and Planning is funded in the G\&A Pool. Major costs are management, analytical, and clerical iabor support; office supplies; purchased services such as offsite training and travel; site services such as QTRC training; and BCS maintenance support.

\section{TECHNICAL CONTENT}

Financlal Integration \& Planning provides management direction and leadership for activities related to the mission and objectives of the Controller's Department. Functions include overseeing/coordinating all WHC budgetary/manpower planning and monitoring the execution of all obligated funds to insure effective utilization and control. Activities comply with current applicable regulations and guidance as outlined in the DOE-RL contract, DOE Orders and Directives, Labor Contracts, WHC Management Requirements and Procedures, Management Policies and Manuals.

\section{OBJECTIVES - (Specific)}

- Coordinate and integrate all program (EM, excluding Five-Year Plan and MYPP, and non-EM) budget submissions and related special company level exercises to ensure compliance with financial and budgetary regulations.

- Prepare responses to requests for budgetary information, impact analysis, and development of briefing material. Coordinate budget scenarios as required.

- Maintain company-level tracking of all current year programmatic funding, fiscalyear spending forecasts, commitments and year-end trending (i.e., trending during the year as well as year-to-year comparison).

- Coordinate impact analysis and recommend resolution of funding issues which arise during the execution year for all areas budgeted (e.g., prioritization lists, reprogramming and supplemental requests, budget information to satisfy "funding" requirements of Paragraph 139, Section D of the Tri-Party Agreement.

- Ensure timely commitment and monitoring of capital equipment and GPP funds.

- Development and maintenance of an approved budget formulation manual that includes documentation standards.

- Conduct independent analysis to assure proper documentation and adequate justification to support budget requests in order to allow for independent and/or customer validation.

\section{OBJECTIVES =_(General)}

- Conduct all operations in a sate and cost-efficient manner.

- Meet RL Budget Division requirements and commitments.

- Manage all activities in a total quality manner.

- Develop and maintain policies and procedures to ensure effective internal controls for all funds obligated under the M\&O contract.

- Provide oversight/coordination of all WHC budgetary activities.

- Coordinate miscellaneous budget schedules and crosscuts as required in the annual Unified Budget Call issued by RL Budget Division.

- Develop and maintain company-level funding profiles; and prepare responses involving budgetary data (e.g., funding and manpower) as requested by RL Management, DOE-HQ, OMB, Congress, the Media, and/or the public. This includes coordinating manpower projections with Human Resources.

- Develop/coordinate the preparation of budget briefings to RL/HQ/State/EPA/etc..

- Preparation of other miscellaneous requirements (e.g., Outlays, FIVRS, Reimbursable Work, Overhead/Indirect activity pool status, monthly SMS reporting, manpower analysis and reporting, CFO biweekly, and AFP/DISCAS/SMS reconciliation. 


\begin{tabular}{|c|c|c|}
\hline $\begin{array}{l}\text { COS: } \\
1 \mathrm{MDD}:=\end{array}$ & & \\
\hline $\begin{array}{l}\text { Work Breakdown } \\
\text { Structure } \\
\text { Dictionary }\end{array}$ & $\begin{array}{c}\text { Westinghouse Hanford Company } \\
\text { 6.10.1 BUDGET } \\
\text { Part II - Element Definition }\end{array}$ & $\begin{array}{l}\text { FY } 1995 \text { SSPP } \\
\text { Revision \# } 0\end{array}$ \\
\hline
\end{tabular}

\begin{tabular}{l}
\hline WBS ELEMENT CODE: $\quad 6.10 .1 .1$ \\
$\begin{array}{l}\text { ELEMENT TASK DESCRIPTION } \\
\text { OBJECTIVES - (General continued) }\end{array}$ \\
- Conduct analysis of uncosted obligations for budget fomulation certification to ensure balances are accurately reflected and are considered in the \\
development of requests for new budget authority.
\end{tabular}

development of requests for new budget authority.

- Conduct analysis of uncosted obligations.

- Develop and maintain procedures and funding controls to ensure effective startup and closeout of each fiscal year.

- Ensure that all staff responsible for preparing or reviewing program and indirect budgets are trained to adhere to proper financial and budgetary standards.

- Evaluate the effectiveness of the internal program budget development process to ensure that well reasoned, defendable budgets are being produced for EM and non-EM activities.

- Perform activities to ensure effective utilization and proper control and execution of all obligated funds, including monitoring fiscal year spending forecasts.

- Provide adequate oversight through surveillances and reviews, and internal audits based on an analysis of audit risks.

\section{ASSUMPTIONS/CONSTRAINTS -}

- Budget schedules for EM Programs must be consistent with requirements identified in the ADS, as modified by subsequent change requests.

- Budget submissions for non-EM only programs must be consistent with the Unified Budget Call and associated program guidance.

- Program budget funding baselines should be consistent with FY funding levels as identified in guidance received from RL. Budget Division. Proposed supplemental/reprogramming requests to cover identified shortfalls should not be assumed in developing the program budget funding baselines.

- Coordination of activities between Financial Integration \& Planning and Program Baseline Integration is assumed.

- Activities must comply with current applicable regulations and guidance: Federalstate Regulations; DOE Contract; DOE Orders and Directives; DOE/RL

Guidance/Correspondence; Labor Contracts; WHC Management Guidance; Management Requirements and Procedures (MRP), Management Policies and Othe Controlled Manuals.

\section{MILESTONES -}

- Systematic Uncosted Obligations Analyses

- Outlay Estimate Revisions

- Quarterly Outlay Variance Report

- Internal Evaluation/Surveillance Schedule, Reports, and Corrective Action Plans

- FIVRS Cost Estimate

- Monthly Financial CFO Briefing Report

\section{DELIVERABLES -}

- Monthly Site Management System Report

- FY 1996 SSPP Draft and Final Report

- Unified Budget Call Exhibits

- Budget Formulation/Execution Manual Draft and Final Report

- Transfers To/From Other Hanford Contractors Report 
WORK STATEMENT -

\section{REQUESTS WITHIN TARGET:}

Activity Detailed Description

Financial Integration \& Planning is staffed with one manager. The responsibilities include staffing, management, and leadership to assure the mission and objectives are accomplished within the scheduled milestones and within budgel constraints.

Financial Integration \& Planning is staffed with one full-time secretary to provide secretarial support. Responsibilities include mail distribution, typing, scheduling, report/letter preparation, copying, phone pickup, spreadshee/schedule preparation and general assistance to staff. Secretary workscope transferred from 1 MDD 15 in FY94.

Financial Integration \& Planning is staffed with one full- time clerical staff. Full time clerical responsibilities include Weekly \& Biweekly Staffing Reporting, Corporate Briefing Charts, CFO and SMS reporting, SSPP document preparation, and training support.

Financial Integration \& Planning is slaffed with one part-time CCE clerical staff. Part time clerical responsibilities include Uncosted Analysis input and preparation, Funds Control Table input, analytical support to analysts, and general office support as required.

Financial Integration \& Planning is staffed with 4.9 exempt Project Control Analysts to provide oversight and coordination of all WHC budgetary and manpower planning activities.

One full-time analyst is responsible for reconciling the Funds Control Table, provide an uncosted analysis, provide RL CFO reporting, Expense Summaries, Financial Overview preparation, and special requests as designated.

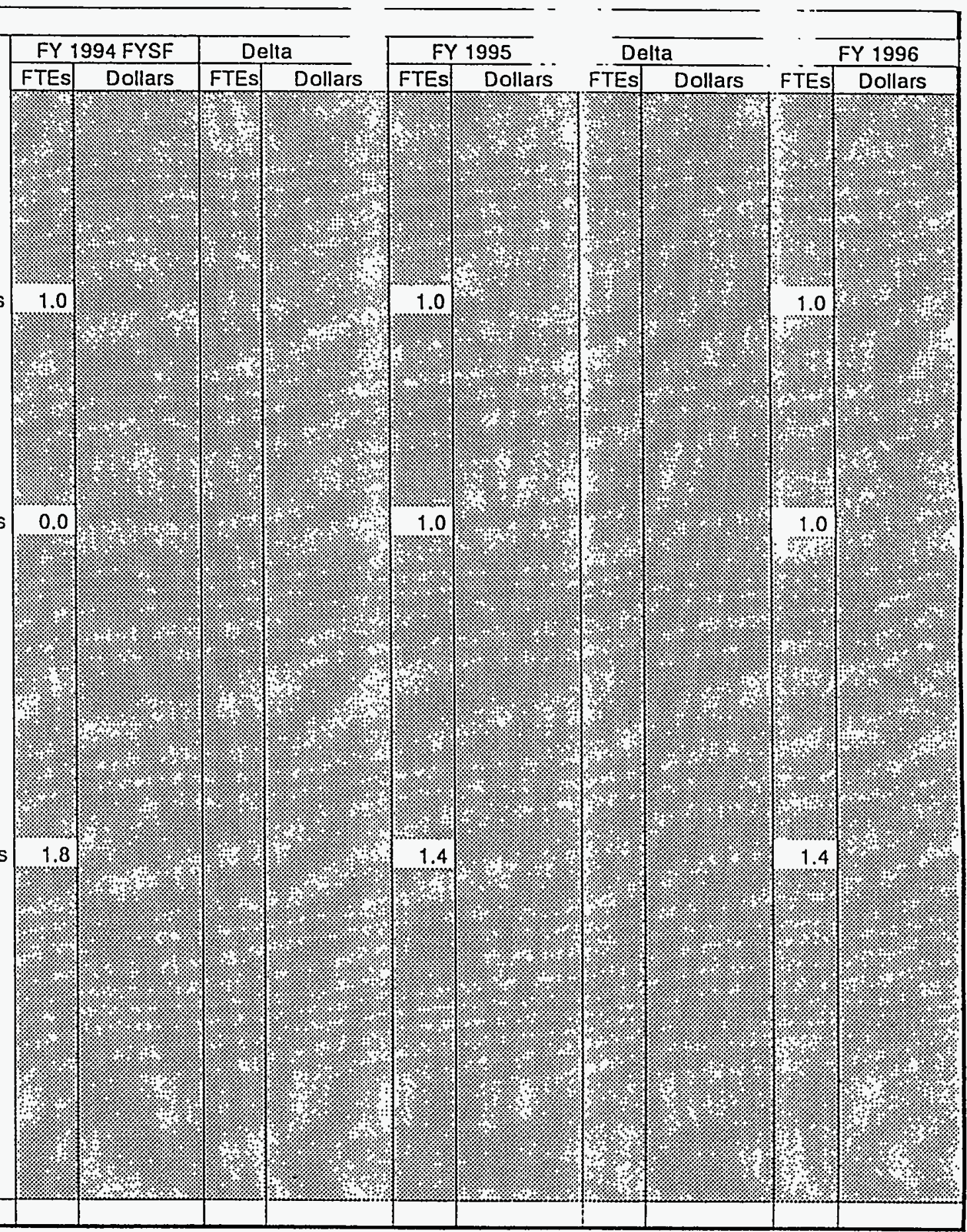




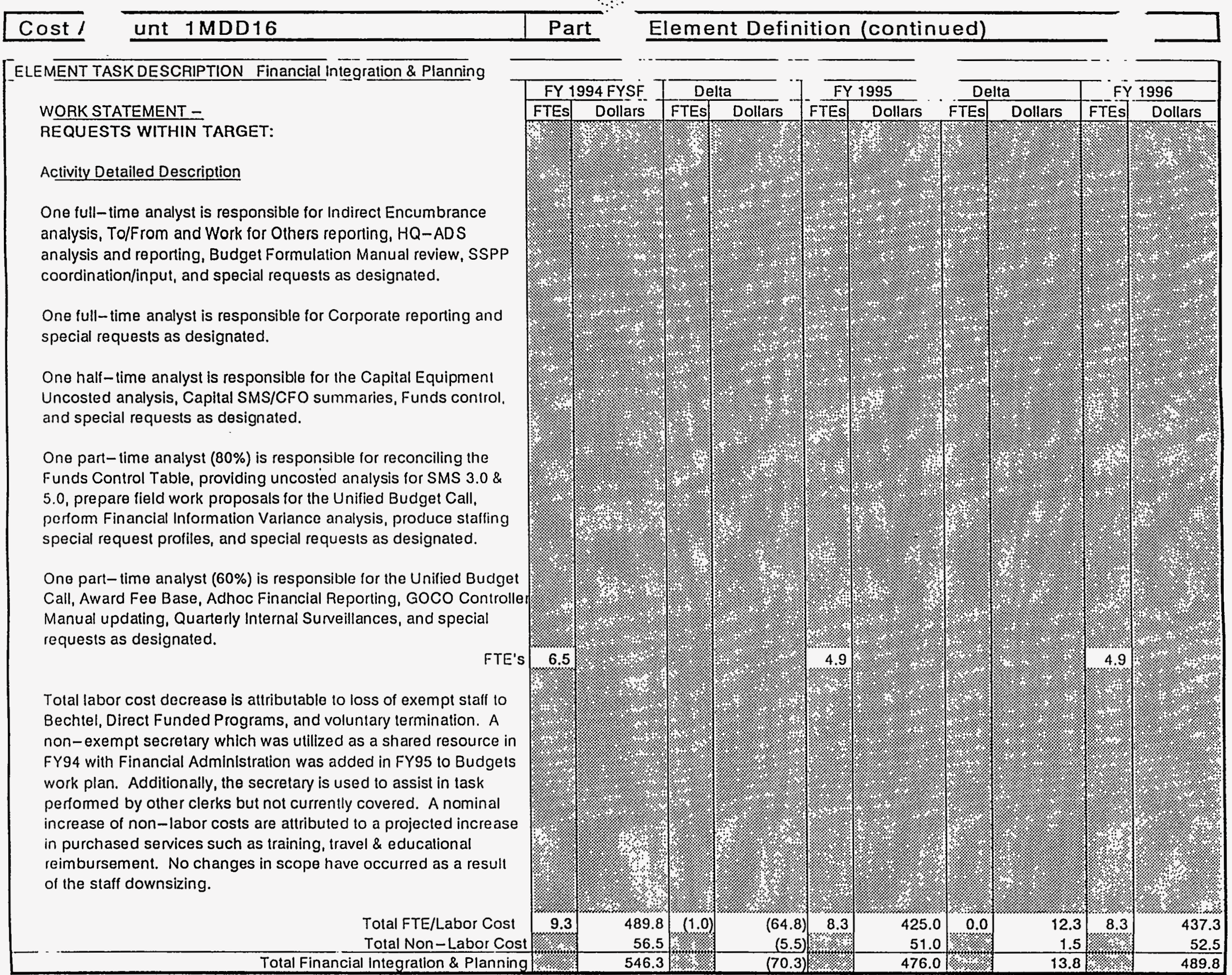




\begin{tabular}{|c|c|c|}
\hline $\begin{array}{ll}\cos & \text { sOUNT } \\
1 \mathrm{MDD}, & \end{array}$ & & \\
\hline $\begin{array}{l}\text { Work Breakdown } \\
\text { Structure } \\
\text { Dictionary }\end{array}$ & $\begin{array}{c}\text { Westinghouse Hanford Company } \\
\text { 6.10.1 BUDGET } \\
\text { Part } \|- \text { Element Definition }\end{array}$ & $\begin{array}{l}\text { FY } 1995 \text { SSPP } \\
\text { Revision \#0 }\end{array}$ \\
\hline
\end{tabular}

\begin{tabular}{|c|c|c|c|}
\hline WBS ELEMENT CODE: & 6.10 .1 .2 & TITLE: & Financial Services \\
\hline
\end{tabular}

COST CONTENT -

The Financial Services organization contains labor funded both by direct programs and by the G\&A pool. Labor supporting the Overhead/ESQ organization is funded directly by the organization to which service is provided. Management and analyts supporting the Controller's organizatlon in this work plan is funded through the G\&A overhead pool. Major costs are management, analytical, and clerical labor support; office supplies; purchased services such as offsite training and travel; site sercvices such as QTRC training; and BCS maintenance support.

\section{TECHNICAL CONTENT -}

Financial Services provides for overall financial support sevices to company level support organizations including planning, budgeting, forecasting, estimating, analysis, reporting, and maintenance of financial data.

\section{OBJECTIVES}

- Provide overall budget planning and coordination support to the SAS program.

- Conduct all operations in a safe and cost-efficient manner.

- Prepare responses to requests for budgetary information, impact analysis, and development of briefing material.

- Manage all actlvities in a total quality manner.

- Develop and maintain policles and procedures to ensure effective internal controls for designated funds within the specified program.

- Provide oversight/coordination of designated WHC budgetary activities.

\section{ASSUMPTIONS}

- None

\section{MILESTONES}

Quarterly SAS Obligation and Cost Report

\section{DELIVERABLES}

- None 
Westinghouse Hanford Company

MILESTONE DESCRIPTION SHEET

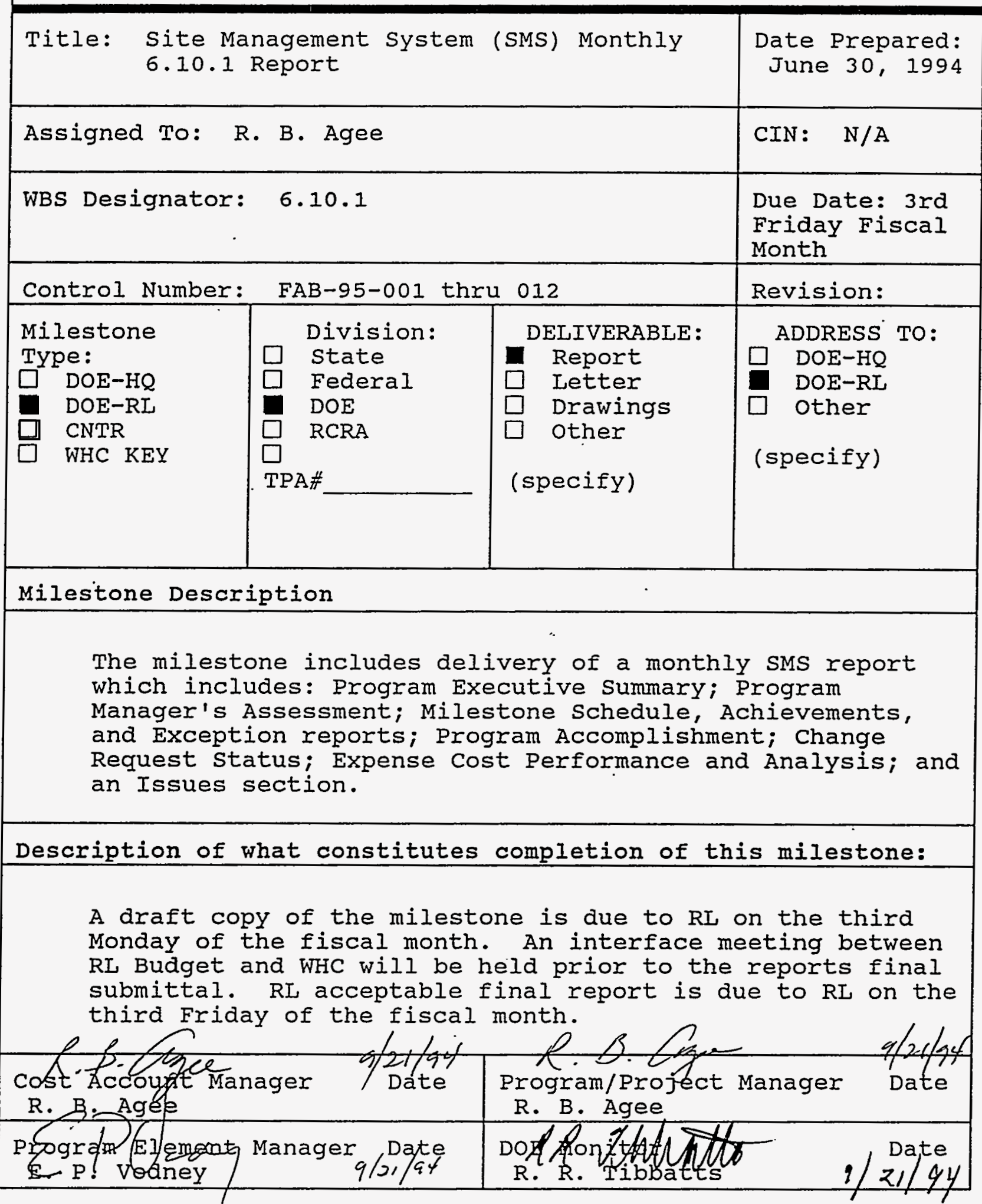




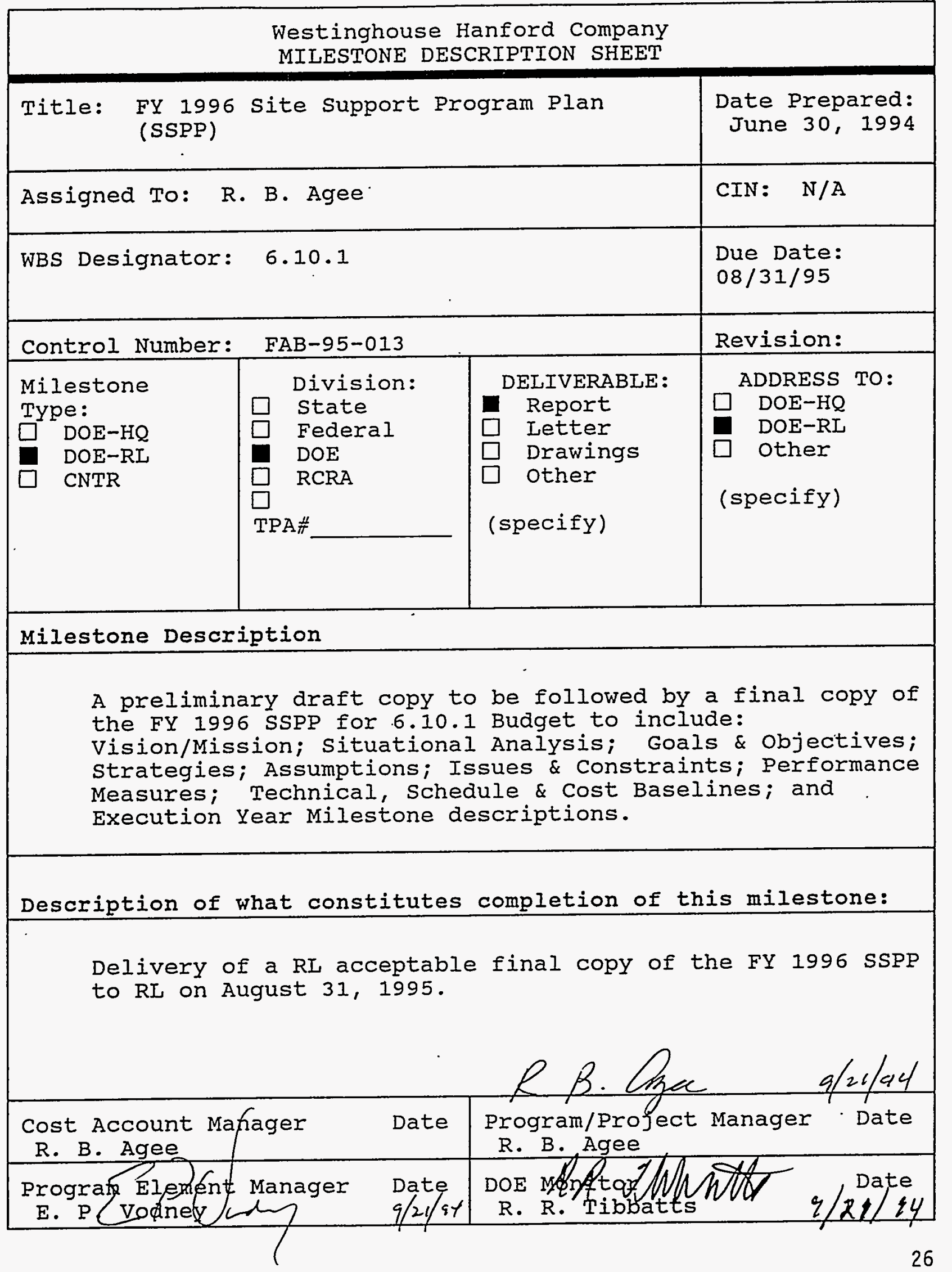




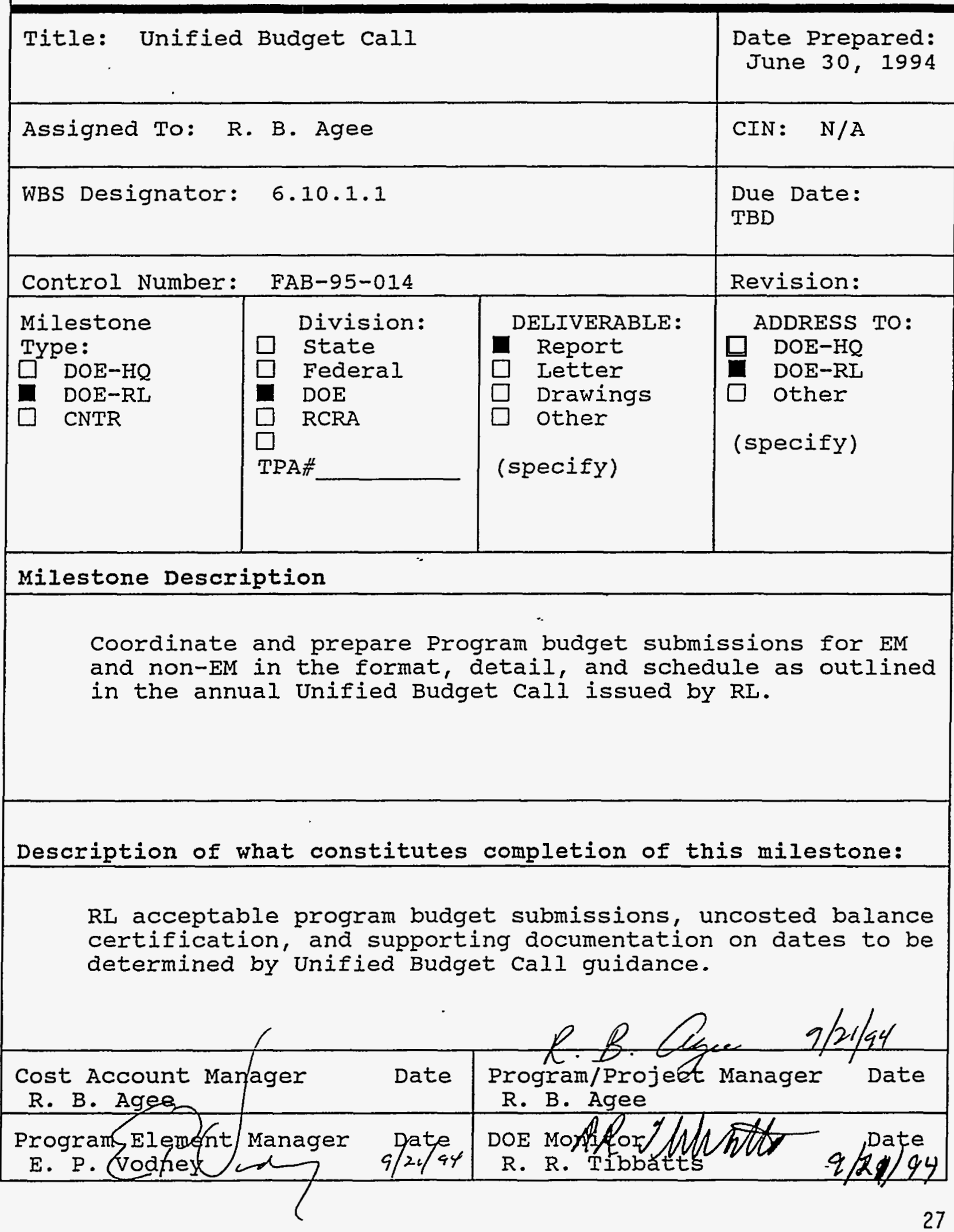




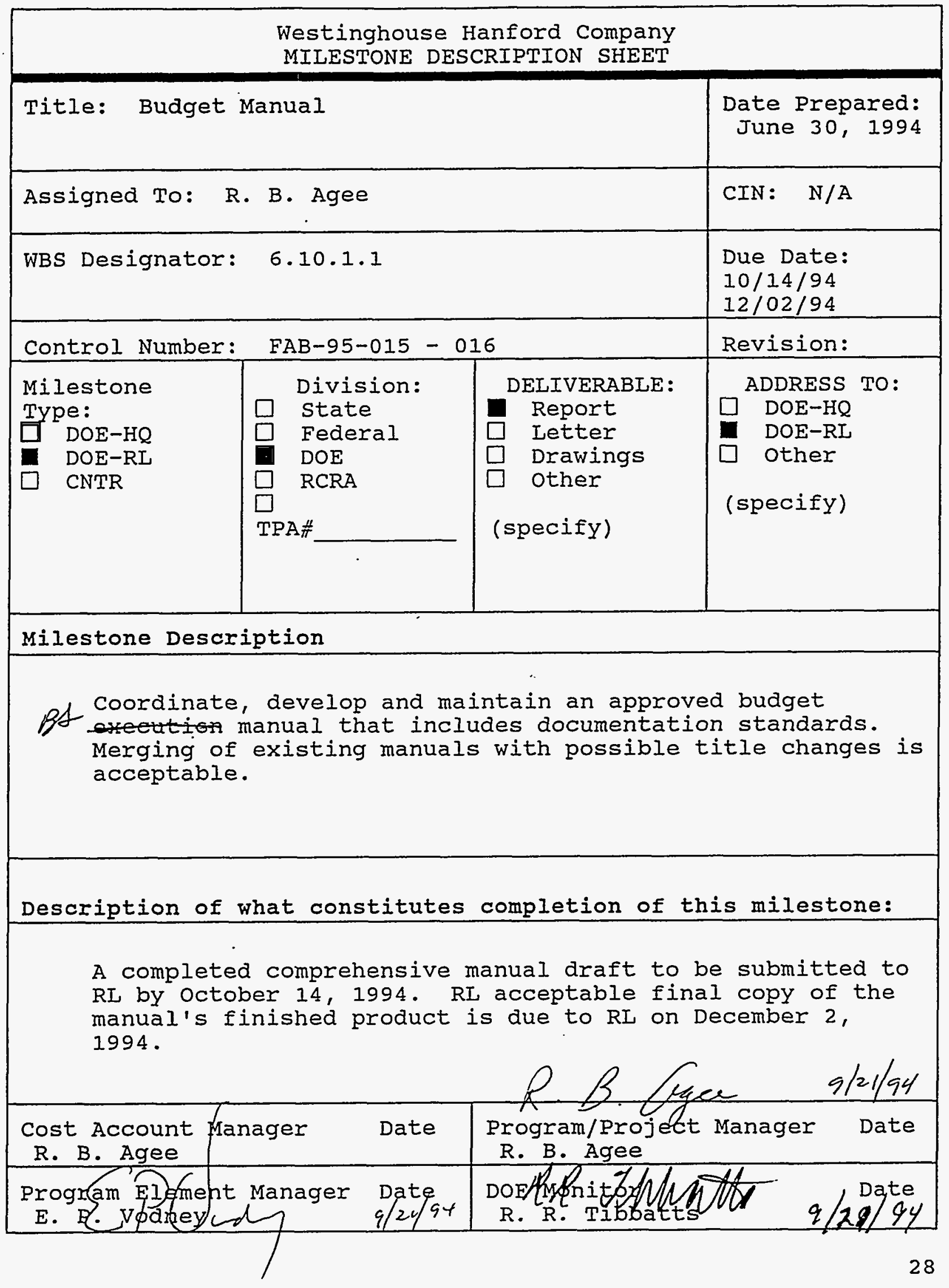




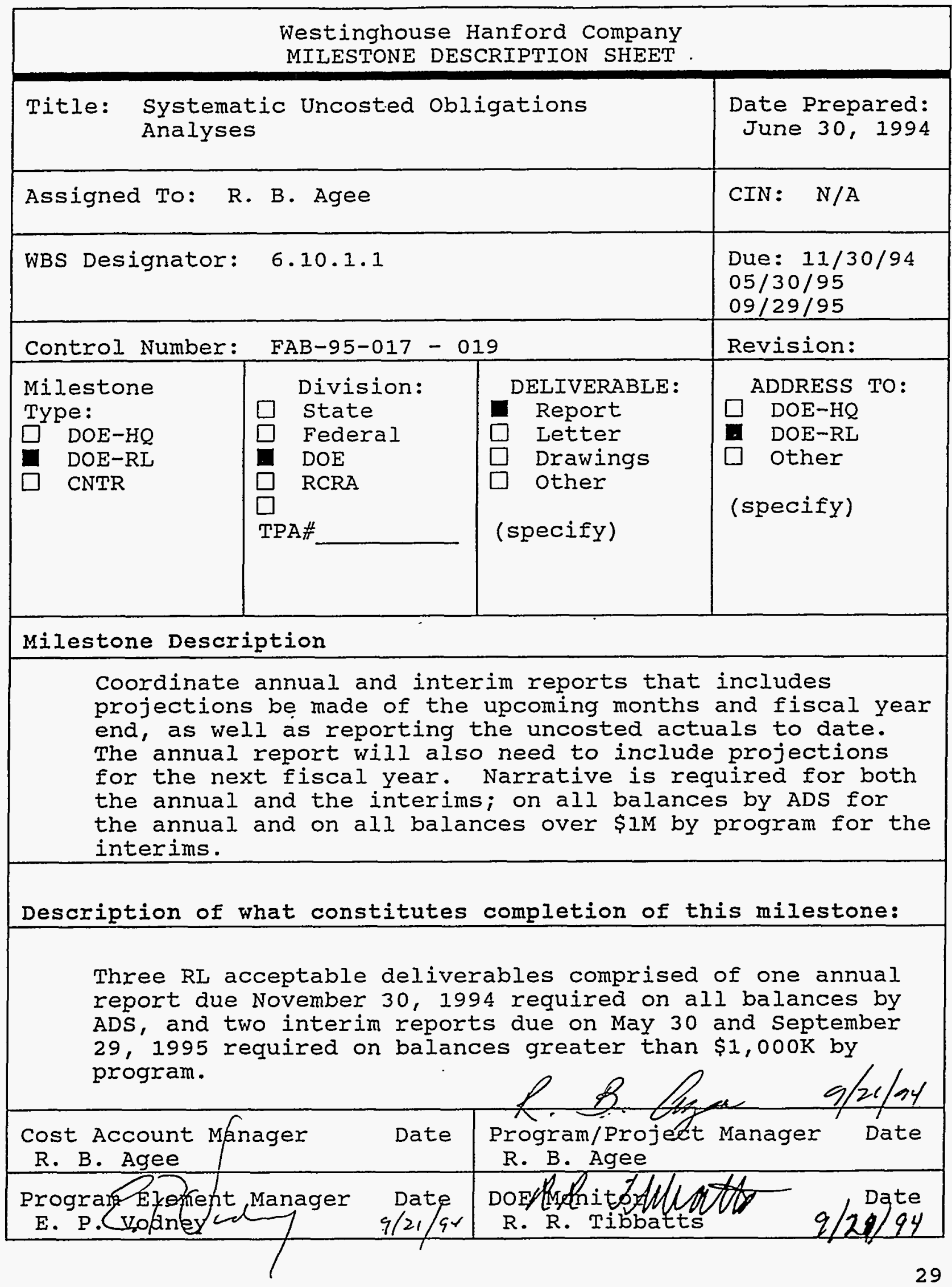


Westinghouse Hanford Company

MILESTONE DESCRIPTION SHEET

\begin{tabular}{|c|c|c|c|}
\hline Title: Uncoste & d Variance An & sis Report & $\begin{array}{l}\text { Date Prepared: } \\
\text { June } 30,1994\end{array}$ \\
\hline Assigned To: R & - B. Agee & & CIN: $\quad N / A$ \\
\hline WBS Designator: & 6.10 .1 .1 & & $\begin{array}{l}\text { Due: } 05 / 19 / 95 \\
06 / 16 / 95 \\
07 / 14 / 95 \\
08 / 18 / 95 \\
09 / 15 / 95 \\
\end{array}$ \\
\hline Control Number: & $\mathrm{FAB}-95-020$ & 024 & Revision: \\
\hline $\begin{array}{l}\text { Milestone } \\
\text { Type: } \\
\square \text { DOE-HQ } \\
\text { DOE-RI } \\
\text { CNTR }\end{array}$ & $\begin{array}{ll} & \text { Division: } \\
& \text { State } \\
\square & \text { Federal } \\
& \text { DOE } \\
\square & \text { RCRA } \\
\square \\
\text { TPA\# }\end{array}$ & $\begin{array}{l}\text { DELIVERABLE: } \\
\text { Report } \\
\square \text { Letter } \\
\square \text { Drawings } \\
\square \text { Other } \\
\text { (specify) }\end{array}$ & $\begin{array}{l}\text { ADDRESS TO: } \\
\square \text { DOE-HQ } \\
\text { DOE-RL } \\
\square \text { Other } \\
\text { (specify) }\end{array}$ \\
\hline
\end{tabular}

Coordinate and develop a report that includes a systematic variance analysis reconciling the monthly spending plans to actual costs. The analysis is per the outline in the RL guidance letter for the budget execution year.

Description of what constitutes completion of this milestone:

Provide RL with acceptable narrative explanations of uncosted variances $(+/-10 \%)$ the last six months of the year. The narrative will be due the fifteenth working day of the following month.

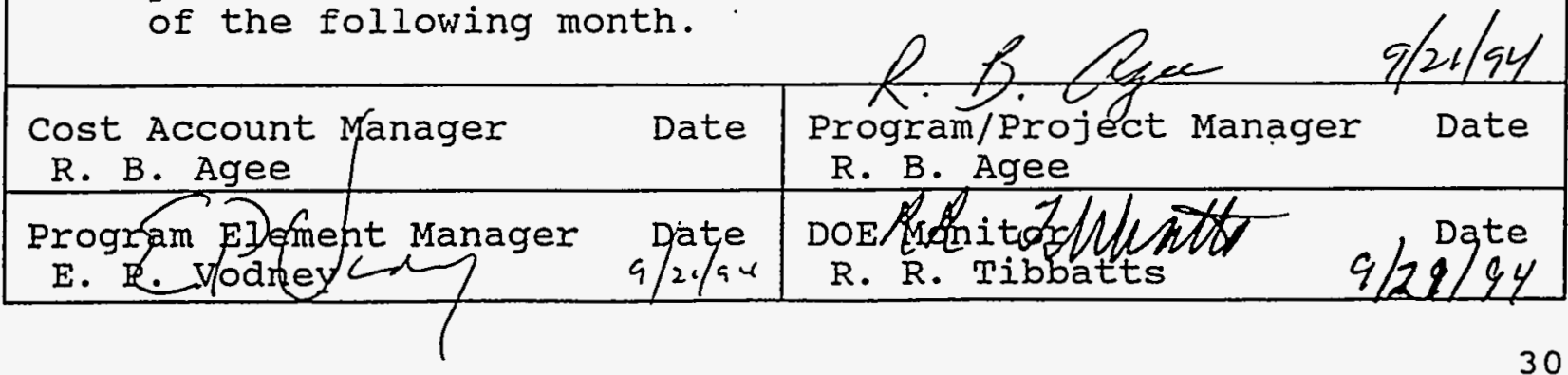


Title: FY 1995 outlay Estimate Revisions and Variance Reports (as needed)

Date Prepared: June 30,1994

Assigned To: R. B. Agee

CIN : $N / A$

WBS Designator: 6.10 .1 .1

Due: $12 / 06 / 94$

$03 / 07 / 95$

$06 / 06 / 95$

Control Number: FAB-95-025 thru 027

Revision:

Milestone

Type:

DOE-HQ

DOE-RL

CNTR

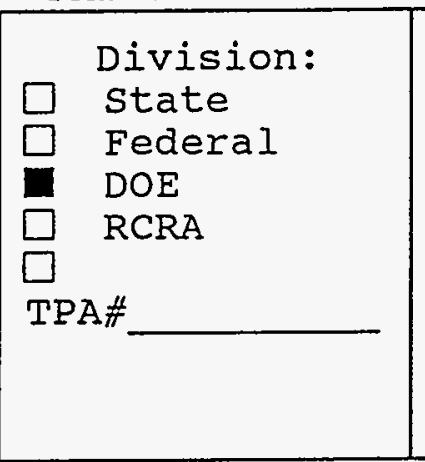

DELIVERABLE :

Report

ADDRESS TO:

Letter

$\square$ Drawings

other

(specify)

DOE-HQ

- DOE-RL

$\square$ other

(specify)

\section{Milestone Description}

Coordinate and develop outline revisions to the FY 1995 outlay estimates by appropriation. Variance reports to be provided on a quarterly basis only if estimates exceed variance threshold variance guidelines.

Description of what constitutes completion of this milestone:

Three RL acceptable reports constitute completion of this milestone. The first report is to RL December 6, 1994. The second report is due to RL on March 7, 1995 and final report is due to RI June $6,1995$.

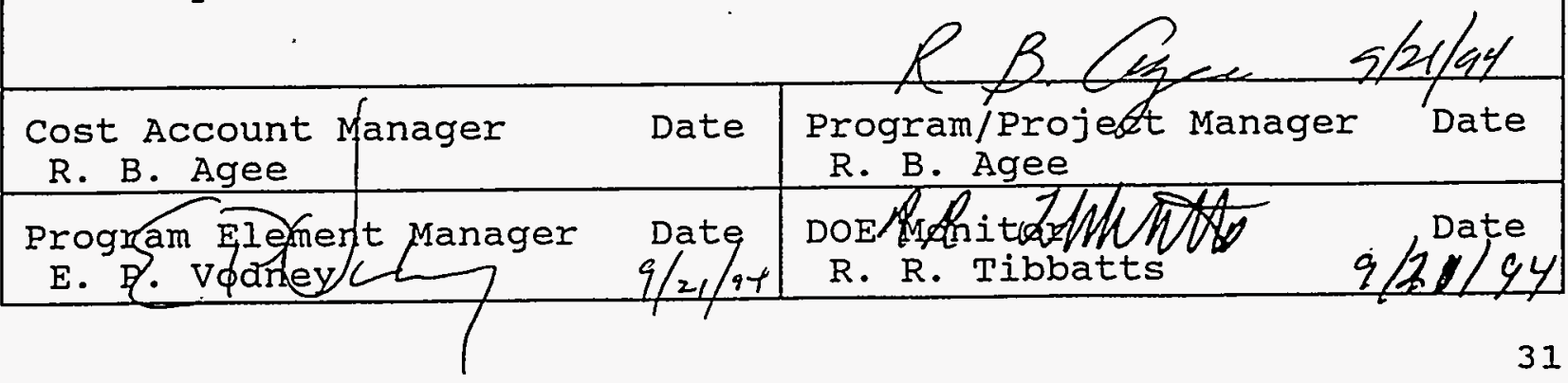




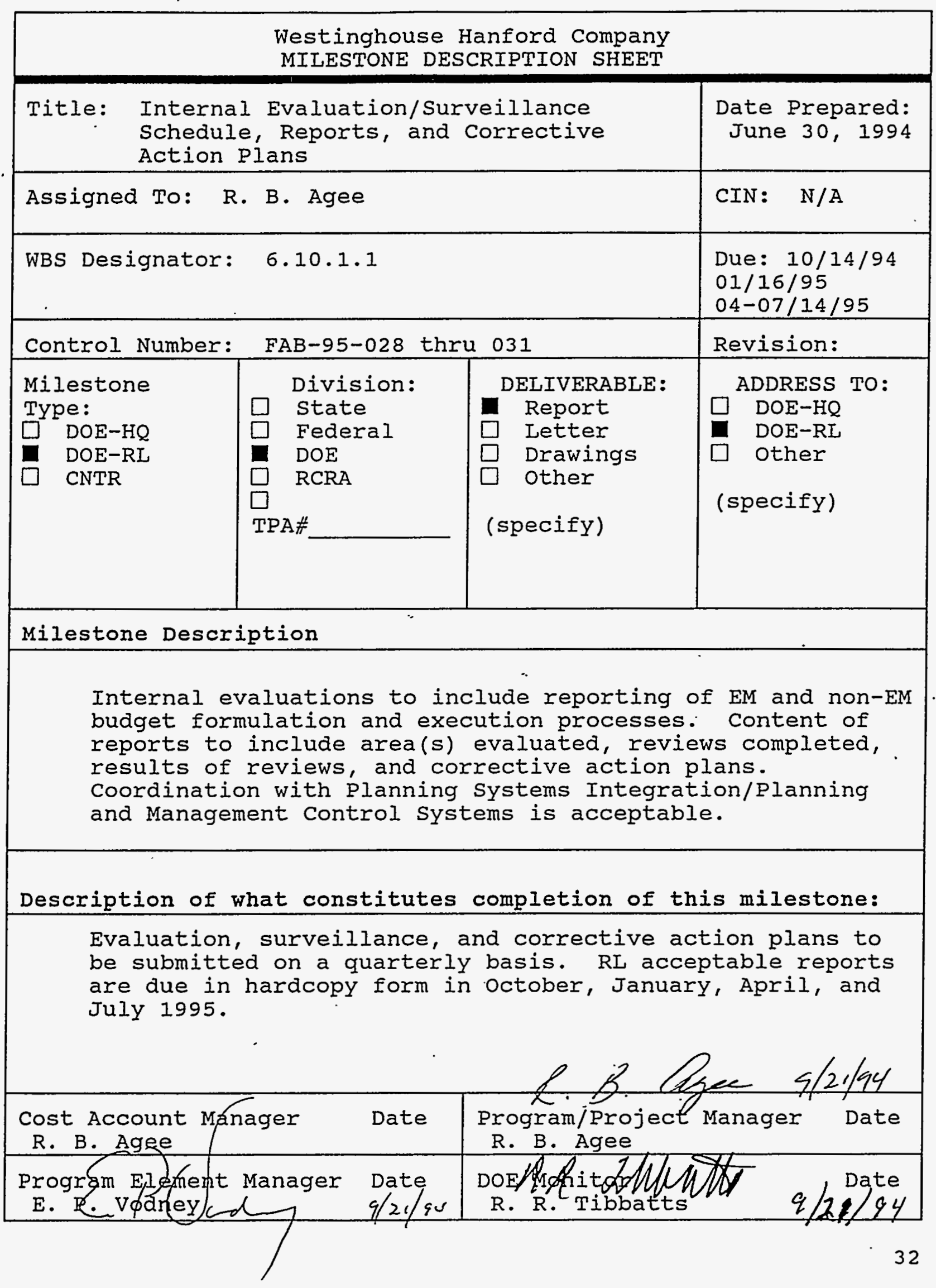




\begin{tabular}{|c|c|c|c|}
\hline \multicolumn{3}{|c|}{ Title: FIVRS cost Estimate } & $\begin{array}{l}\text { Date Prepared: } \\
\text { June } 30,1994\end{array}$ \\
\hline \multicolumn{3}{|c|}{ Assigned To: R. B. Agee } & CIN: $\quad N / A$ \\
\hline \multicolumn{3}{|c|}{ WBS Designator: 6.10 .1 .1} & $\begin{array}{l}\text { Due Date: } \\
10 / 14 / 94 \\
04 / 14 / 95\end{array}$ \\
\hline \multicolumn{3}{|c|}{ Control Number: $F A B-95-032-033$} & Revision: \\
\hline $\begin{array}{l}\text { Milestone } \\
\text { Type: } \\
\square \text { DOE-HQ } \\
\text { DOE-RL } \\
\square \text { CNTR }\end{array}$ & $\begin{array}{ll} & \text { Division: } \\
\square & \text { State } \\
\square & \text { Federal } \\
& \text { DOE } \\
\square & \text { RCRA } \\
\square \\
\text { TPA\# }\end{array}$ & $\begin{array}{l}\text { DELIVERABIE: } \\
\quad \text { Report } \\
\square \text { Letter } \\
\square \text { Drawings } \\
\square \text { Other } \\
\text { (specify) }\end{array}$ & $\begin{array}{l}\text { ADDRESS TO: } \\
\square \text { DOE-HQ } \\
\square \text { DOE-RI } \\
\text { Other } \\
\text { (specify) }\end{array}$ \\
\hline
\end{tabular}

FIVRS Cost Estimating provides DOE-RI with monthly cost projections for Defense Program (DP) operating dollars and associated monthly narrative explanations when variances to cost projections are generated. FIVRS reporting occurs semi-annually.

Description of what constitutes completion of this milestone:

Submittal is required on a semi-annual basis. RI acceptable report is required on October 15, 1994 and April 15, 1995.

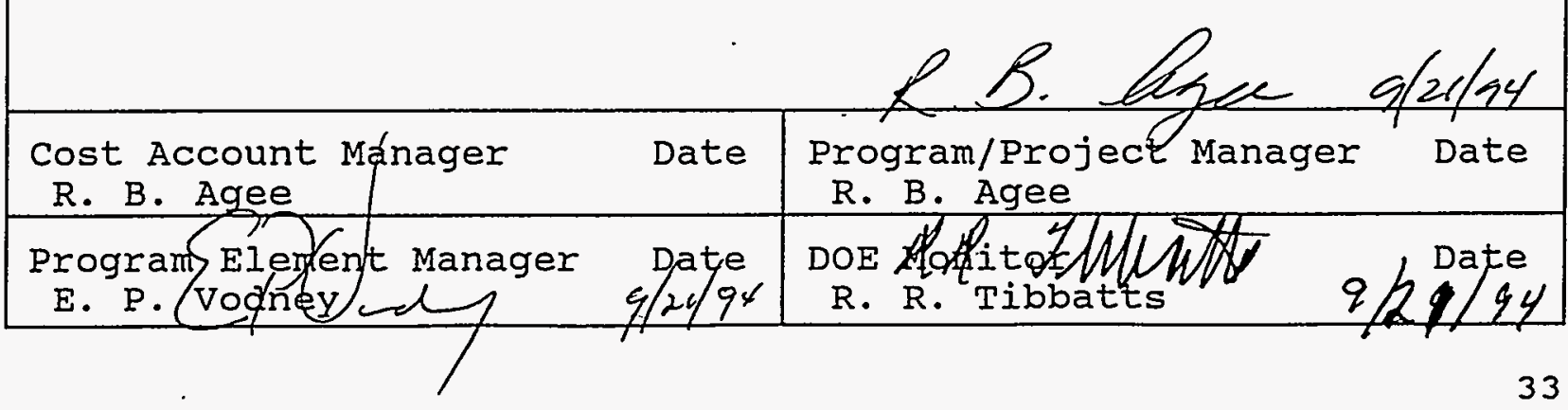




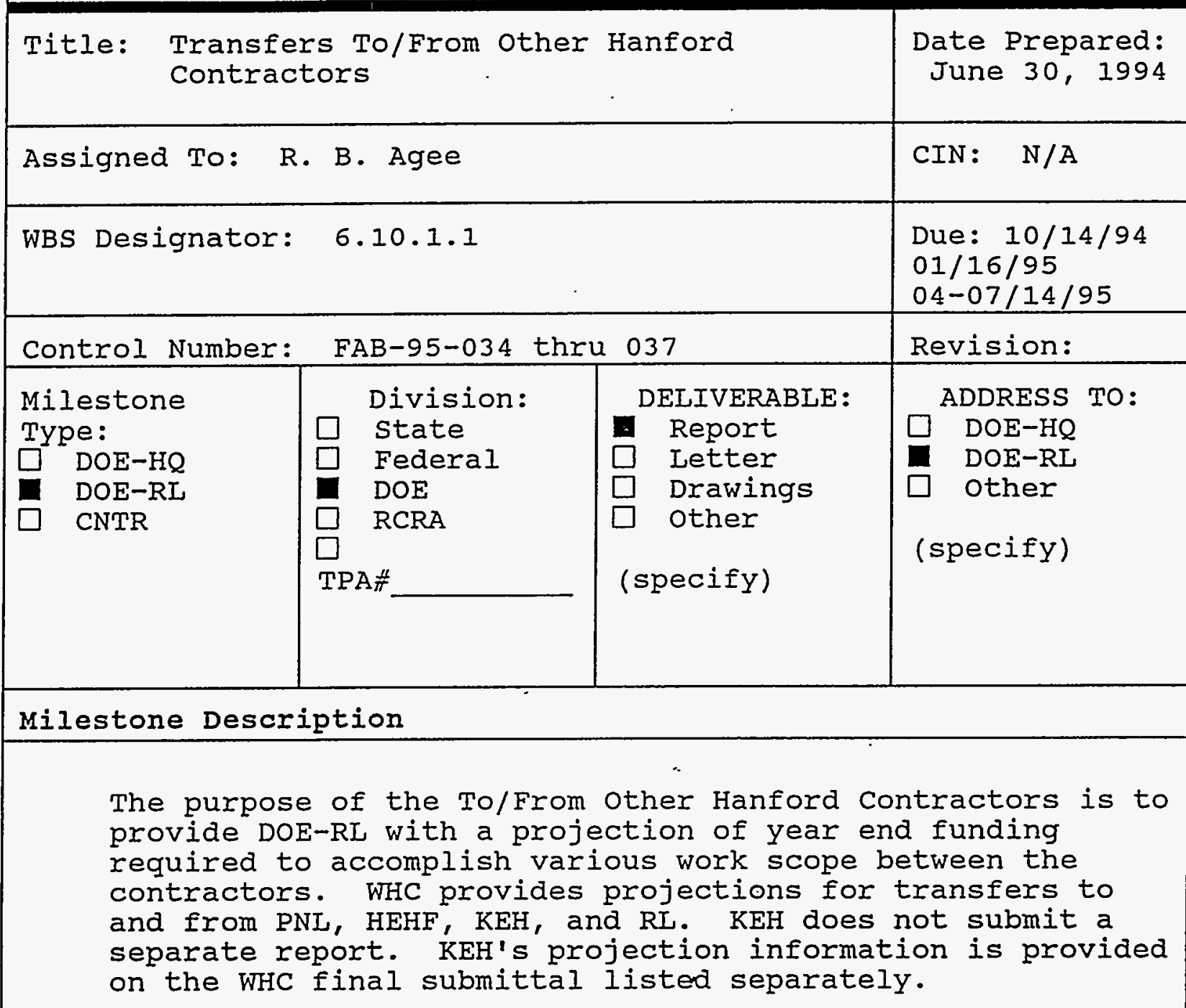

Description of what constitutes completion of this milestone:

RL acceptable hardcopy submittal is required on a quarterly basis. The report is required on october 15, 1994, January, April and July 15, 1995.

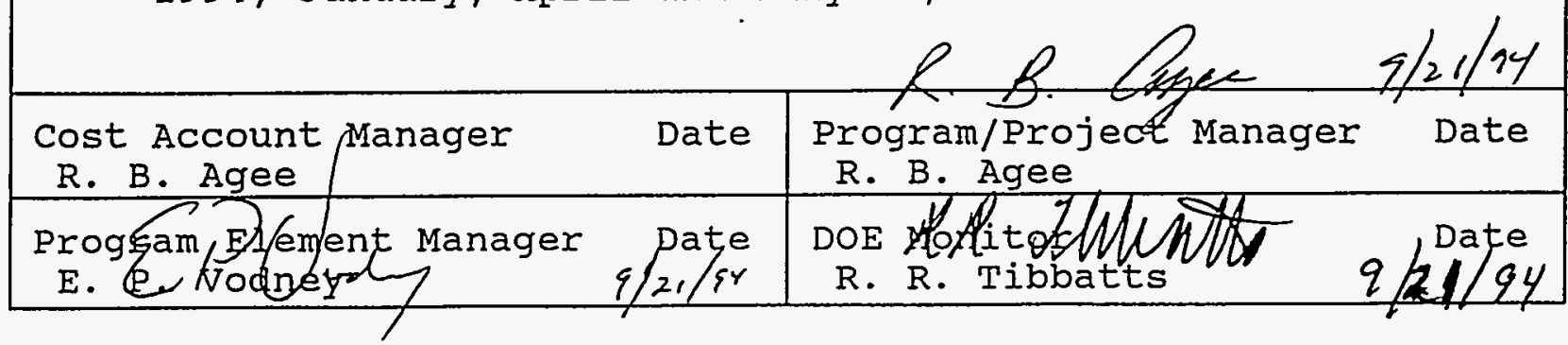




\begin{tabular}{|c|c|c|c|}
\hline Title: Monthly & Budget CFO Brie & ing Report & $\begin{array}{l}\text { Date Prepared: } \\
\text { June } 30,1994\end{array}$ \\
\hline Assigned To: $R$ & - B. Agee & & CIN: $\quad N / A$ \\
\hline WBS Designator: & 6.10 .1 .1 & & Due Date: TBD \\
\hline Control Number: & FAB-95-038 thre & 049 & Revision: \\
\hline $\begin{array}{l}\text { Milestone } \\
\text { Type: } \\
\square \text { DOE-HQ } \\
\text { DOE-RL } \\
\square \text { CNTR }\end{array}$ & $\begin{array}{ll} & \text { Division: } \\
\square & \text { State } \\
\square & \text { Federal } \\
& \text { DOE } \\
\square & \text { RCRA } \\
\square & \\
\text { TPA\# }\end{array}$ & $\begin{array}{l}\text { DELIVERABLE: } \\
\square \text { Report } \\
\square \text { Letter } \\
\square \text { Drawings } \\
\text { other } \\
\\
\text { (specify) } \\
\text { Briefing }\end{array}$ & $\begin{array}{l}\text { ADDRESS TO: } \\
\square \text { DOE-HQ } \\
\text { DOE-RI } \\
\square \text { Other } \\
\text { (specify) }\end{array}$ \\
\hline
\end{tabular}

The monthly Financial Briefing to RL's CFO is an overall company level briefing. The briefing includes capital commitments and expense funding status detailing burn rates and spending forecasts by program. The briefing also serves as a formal means to address issues and concerns.

Description of what constitutes completion of this milestone:

RL acceptable report as well as a briefing is required on a monthly basis on a pre-set schedule to accomplish this milestone.

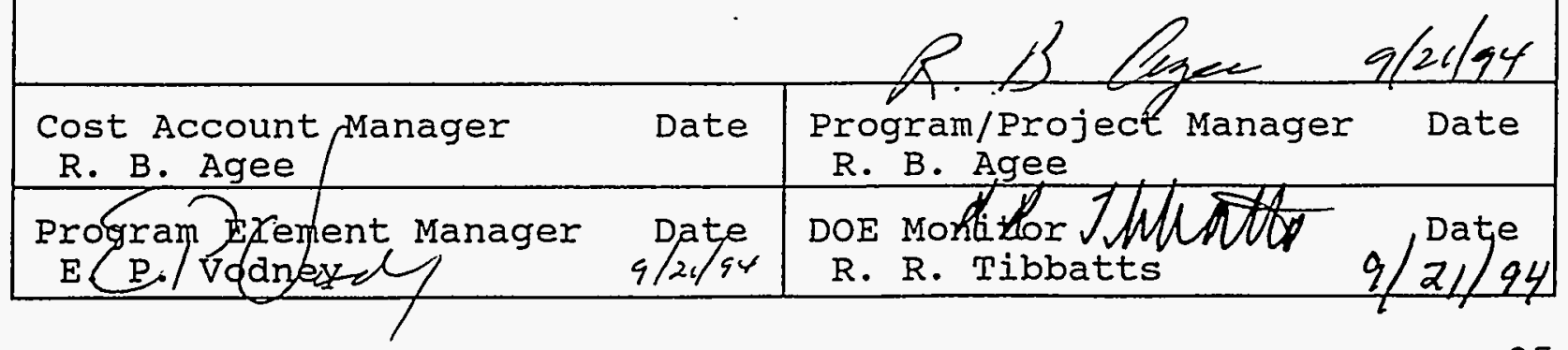


Title: SAS Obligation and cost Report

Date Prepared:

June 30,1994

Assigned To: I. H. Peterson

CIN: $N / A$

WBS Designator: 6.10 .1 .2

Due: $10 / 14 / 94$

$01 / 16 / 95$

$04-07 / 14 / 95$

Control Number: FAB-95-050 thru 053 Revision:

Milestone

Type:

$\square$ DOE-HQ

DOE-RL

CNTR

Division:

State

Federal

DOE

RCRA

TPA\#
DELIVERABLE :

a Report

Letter

Drawings

other

(specify)

Briefing
ADDRESS TO:

DOE-HQ

DOE-RL

Other

(specify)

Milestone Description

The SAS obligation and cost report provides a status of SAS activities against "planned estimates". The FY.1995 SAS Budget Estimates (Crosscut) for operating expense, capital equipment, general plant projects, and line item construction projects as submitted are used for report preparation.

Description of what constitutes completion of this milestone:

RL acceptable quarterly sAS obligations and cost report provided per guidance during first quarter of the fiscal year. Report submitted to Laboratory and overhead Branch.

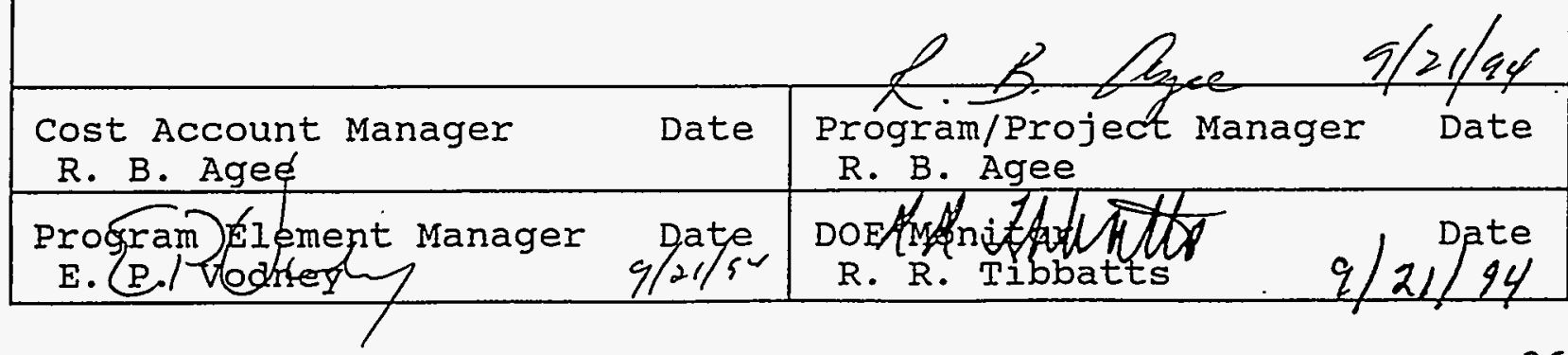




\begin{tabular}{|c|c|c|c|}
\hline \multicolumn{3}{|c|}{$\begin{array}{l}\text { Overhead Planning Self-Surveillance } \\
\text { for Improvements to the FY } 1995 \\
\text { Indirect Planning Process }\end{array}$} & $\begin{array}{l}\text { Date Prepared: } \\
\text { June } 30,1994\end{array}$ \\
\hline \multicolumn{3}{|c|}{ Assigned To: B. R. Thomas } & CIN: $\quad N / A$ \\
\hline \multicolumn{3}{|c|}{ WBS Designator: 6.10 .1 .3} & $\begin{array}{l}\text { Due Date: } \\
07 / 31 / 95\end{array}$ \\
\hline \multicolumn{3}{|c|}{ Control Number: FAB-95-054 } & Revision: \\
\hline $\begin{array}{l}\text { Milestone } \\
\text { Type: } \\
\square \text { DOE-HQ } \\
\text { DOE-RI } \\
\square \text { CNTR }\end{array}$ & $\begin{array}{ll} & \text { Division: } \\
\square & \text { State } \\
\square & \text { Federal } \\
\text { DOE } \\
\square \text { RCRA } \\
\square \text { TPA\# } \\
\end{array}$ & $\begin{array}{l}\text { DELIVERABLE: } \\
\quad \text { Report } \\
\square \text { Letter. } \\
\square \text { Drawings } \\
\square \text { Other } \\
\text { (specify) }\end{array}$ & $\begin{array}{l}\text { ADDRESS TO: } \\
\square \text { DOE-HQ } \\
\text { DOE-RI } \\
\text { Other } \\
\square \\
\text { (specify) }\end{array}$ \\
\hline \multicolumn{4}{|c|}{ Milestone Description } \\
\hline $\begin{array}{l}\text { Provide a } \\
\text { surveillan } \\
\text { and execut } \\
1996 \text { are } t \\
\text { minutes ar } \\
\text { the findin }\end{array}$ & $\begin{array}{l}\text { report detail } \\
\text { ce of the FY } \\
\text { ion processes } \\
\text { o be included } \\
\text { e an acceptab. } \\
\text { gs. }\end{array}$ & $\begin{array}{l}\text { internal evalu } \\
\text { indirect budg } \\
\text { orrective acti } \\
\text { hin evaluation } \\
\text { orm of documen }\end{array}$ & $\begin{array}{l}\text { ion and/or } \\
\text { formulation } \\
\text { plans for FY } \\
\text { OSBRB meeting } \\
\text { tion to support }\end{array}$ \\
\hline
\end{tabular}

Description of what constitutes completion of this milestone:

A hardcopy report is due to RL-Laboratory and Overhead Branch on July 31, 1995.

\begin{tabular}{|c|c|c|c|}
\hline $\begin{array}{l}\text { Cost Account/Manager } \\
\text { B. R. Thoma/s }\end{array}$ & Date & $\begin{array}{l}\text { Program/Project Manager } \\
\text { B. R. Thomas }\end{array}$ & Date \\
\hline 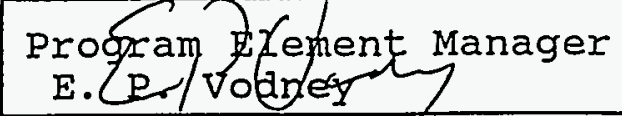 & Date/gu & 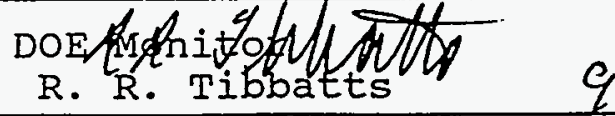 & \\
\hline
\end{tabular}


Westinghouse Hanford Company MILESTONE DESCRIPTION SHEET

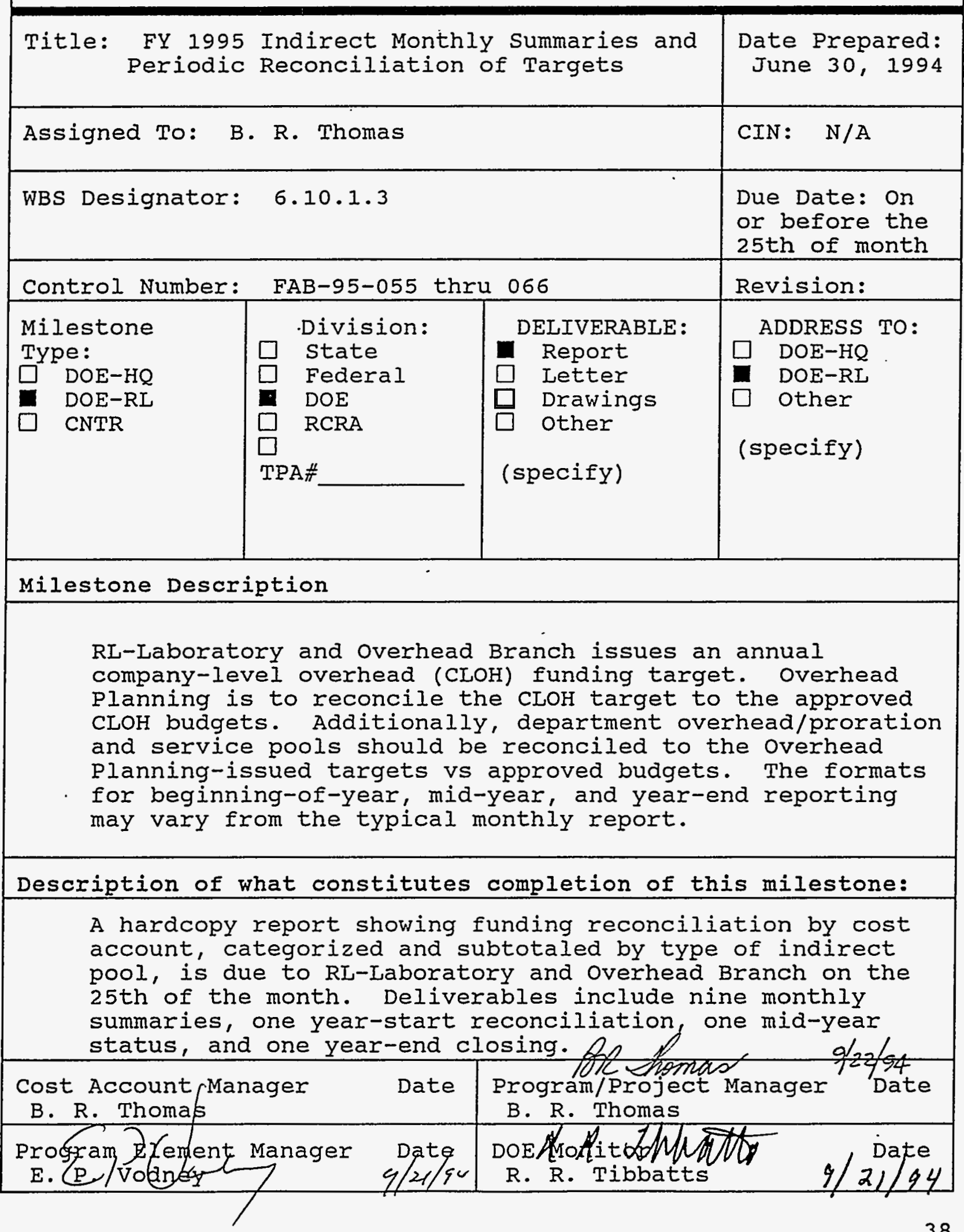




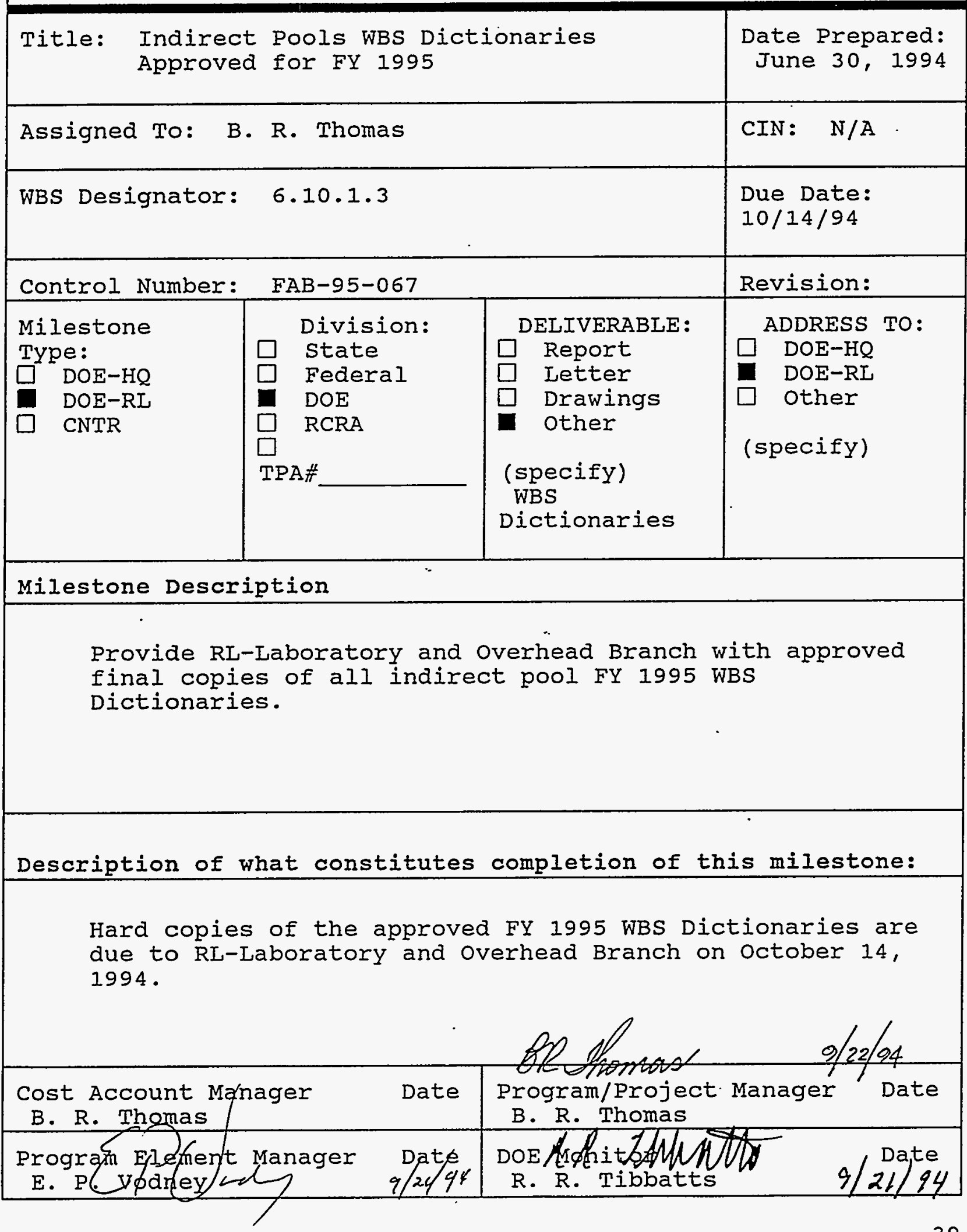




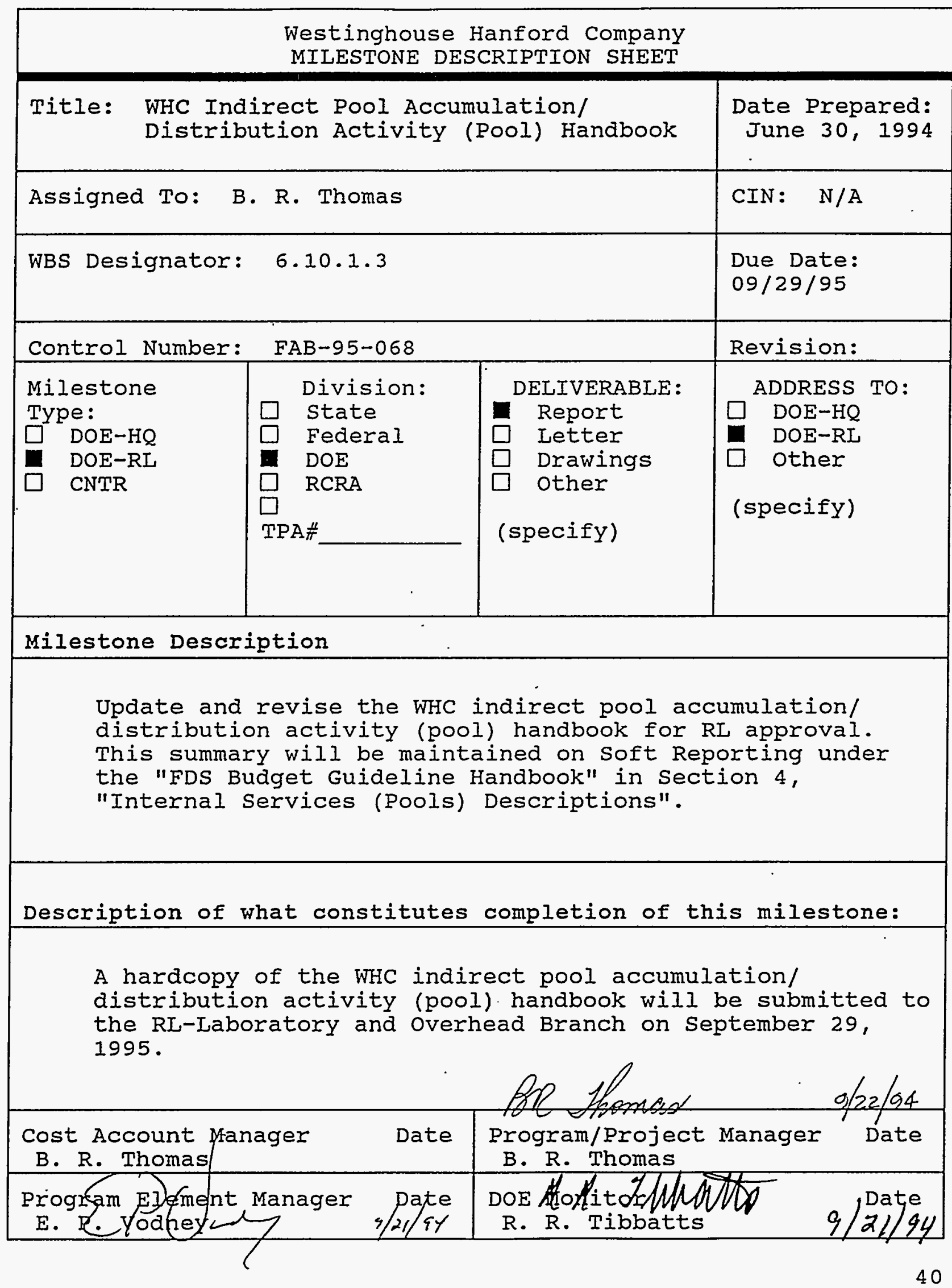




\begin{tabular}{|c|c|c|c|}
\hline \multicolumn{3}{|c|}{$\begin{array}{l}\text { Title: Develop outyear Company-Level Overhead } \\
\text { Planning Rates }\end{array}$} & $\begin{array}{l}\text { Date Prepared: } \\
\text { June } 30,1994\end{array}$ \\
\hline \multicolumn{3}{|c|}{ Assigned To: B. R. Thomas } & CIN: $\quad N / A$ \\
\hline \multicolumn{3}{|c|}{ WBS Designator: 6.10 .1 .3} & $\begin{array}{l}\text { Due Date: } \\
12 / 15 / 94\end{array}$ \\
\hline \multicolumn{3}{|c|}{ Control Number: FAB-95-069 } & Revision: \\
\hline $\begin{array}{l}\text { Milestone } \\
\text { Type: } \\
\square \text { DOE-HQ } \\
\text { DOE-RL } \\
\square \text { CNTR }\end{array}$ & $\begin{array}{ll} & \text { Division: } \\
\square & \text { State } \\
\square & \text { Federal } \\
& \text { DOE } \\
\square & \text { RCRA } \\
\square & \\
\text { TPA\# }\end{array}$ & $\begin{array}{l}\text { DEIIVERABLE: } \\
\square \text { Report } \\
\square \text { Letter } \\
\square \text { Drawings } \\
\square \text { other } \\
\text { (specify) }\end{array}$ & $\begin{array}{l}\text { ADDRESS TO: } \\
\square \text { DOE-HQ } \\
\square \text { DOE-RL } \\
\square \text { other } \\
\text { (specify) }\end{array}$ \\
\hline
\end{tabular}

Coordinate and develop a submittal detailing the companylevel overhead (CLOH) planning rates from FY 1996 through FY 2001. Communicate major changes to service pools/ Department overhead/ Proration (does not apply to continuity of service). This is the information traditionally provided to the ADS planning group.

Description of what constitutes completion of this milestone:

Submit a copy of the ADS planning memo for FY 1996-2001 planning rates to RL-Laboratory and overhead Branch on pember 15, 1994. DEC

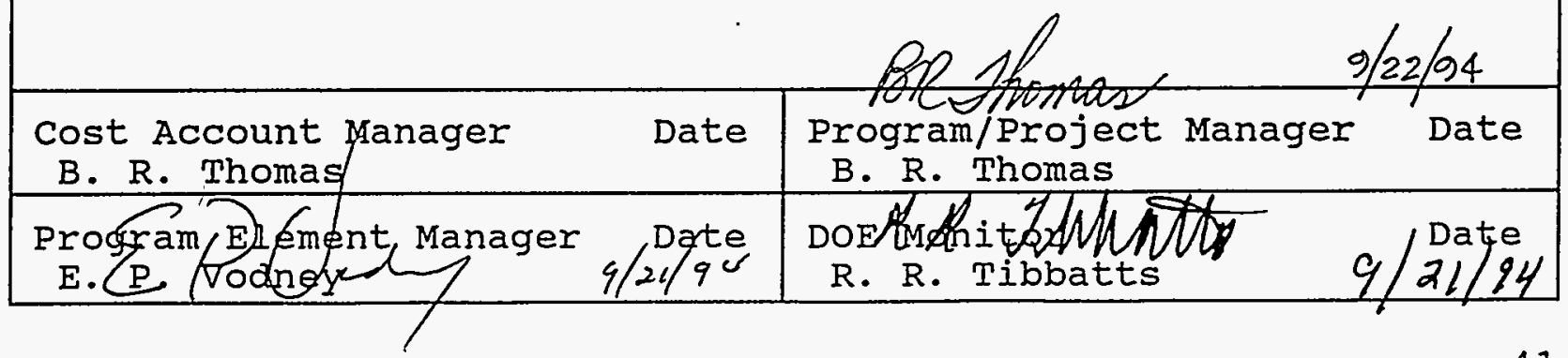




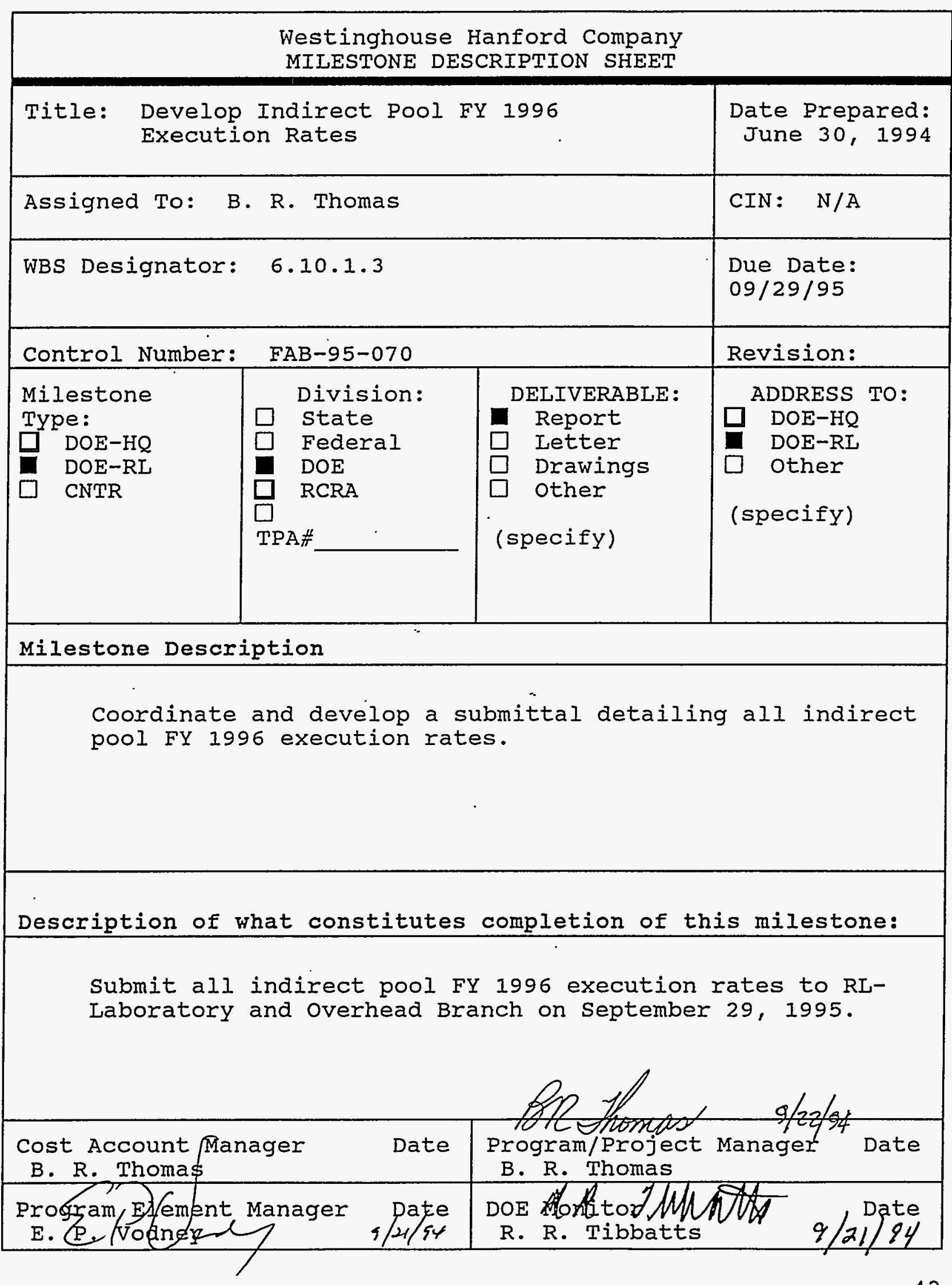




\begin{tabular}{|c|c|c|c|}
\hline Title: $\begin{aligned} \text { Periodi } \\
\text { Reports } \\
\text { and } 6.1\end{aligned}$ & $\begin{array}{l}\text { Site Manage } \\
\text { (Indirect Ex } \\
0.12 \text { ) }\end{array}$ & $\begin{array}{l}\text { System } \\
\text { ive summary }\end{array}$ & $\begin{array}{l}\text { Date Prepared: } \\
\text { June } 30,1994\end{array}$ \\
\hline Assigned To: B & - R. Thomas & & CIN: $\quad N / A$ \\
\hline WBS Designator: & 6.10 .1 .3 & & $\begin{array}{l}\text { Due Date: } 3 \text { rd } \\
\text { Fiscal Friday } \\
\text { of Month }\end{array}$ \\
\hline Control Number: & $F A B-95-071$ & 082 & Revision: \\
\hline $\begin{array}{l}\text { Milestone } \\
\text { Type: } \\
\square \text { DOE-HQ } \\
\text { DOE-RL } \\
\square \text { CNTR }\end{array}$ & $\begin{array}{ll}\square & \text { Division: } \\
\square & \text { State } \\
\square & \text { Federal } \\
\text { DOE } \\
\square \text { RCRA } \\
\square \\
\text { TPA\# }\end{array}$ & $\begin{array}{l}\text { DELIVERABLE: } \\
\text { Report } \\
\square \text { Letter } \\
\square \text { Drawings } \\
\square \text { other } \\
\text { (specify) }\end{array}$ & $\begin{array}{l}\text { ADDRESS TO: } \\
\square \text { DOE-HQ } \\
\text { DOE-RL } \\
\square \text { Other } \\
\text { (specify) }\end{array}$ \\
\hline
\end{tabular}

Coordinate, develop and submit the two periodic indirect SMS reports: the Indirect Executive Summary on a monthly basis and 6.10.12 "Other Miscellaneous Activities" at the end of each quarter.

Description of what constitutes completion of this milestone:

The Indirect Executive Summary is due to RL-Laboratory and overhead Branch on the 3rd fiscal Friday of the month. The 6.10.12 "Other Misc. Accounts" SMS report is due to RL-Laboratory and overhead Branch on october 21, 1994 for FY 1994 closing; January 20, 1995 for lst quarter of FY 1995; April 21 for 2nd quarter; and July 21 for $3 r d$ quarter.

\begin{tabular}{|c|c|c|}
\hline $\begin{array}{l}\text { Cost Account Manager } \\
\text { B. R. Thoma/s }\end{array}$ & Date & $\begin{array}{l}\text { Program/project Manager Date } \\
\text { B. R. Thomas }\end{array}$ \\
\hline $\begin{array}{l}\text { Program Elethent Manager } \\
\text { E.C, Vodney, }\end{array}$ & $\begin{array}{l}\text { Date } \\
\text { g/zI/Gy }\end{array}$ & 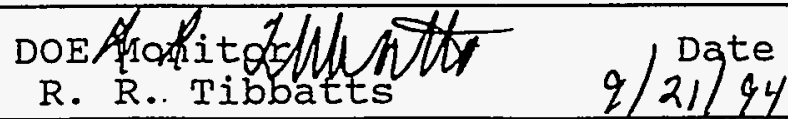 \\
\hline
\end{tabular}




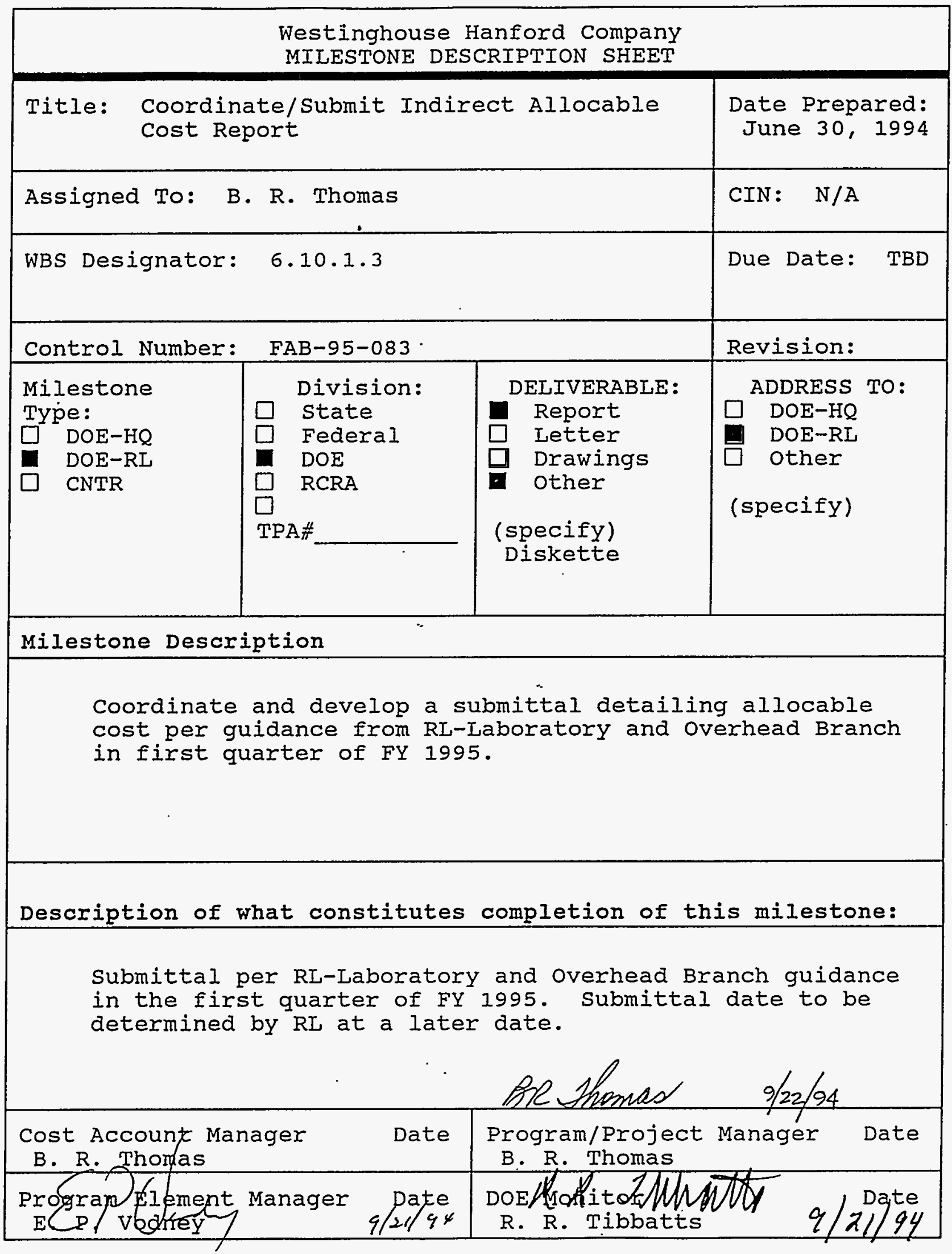




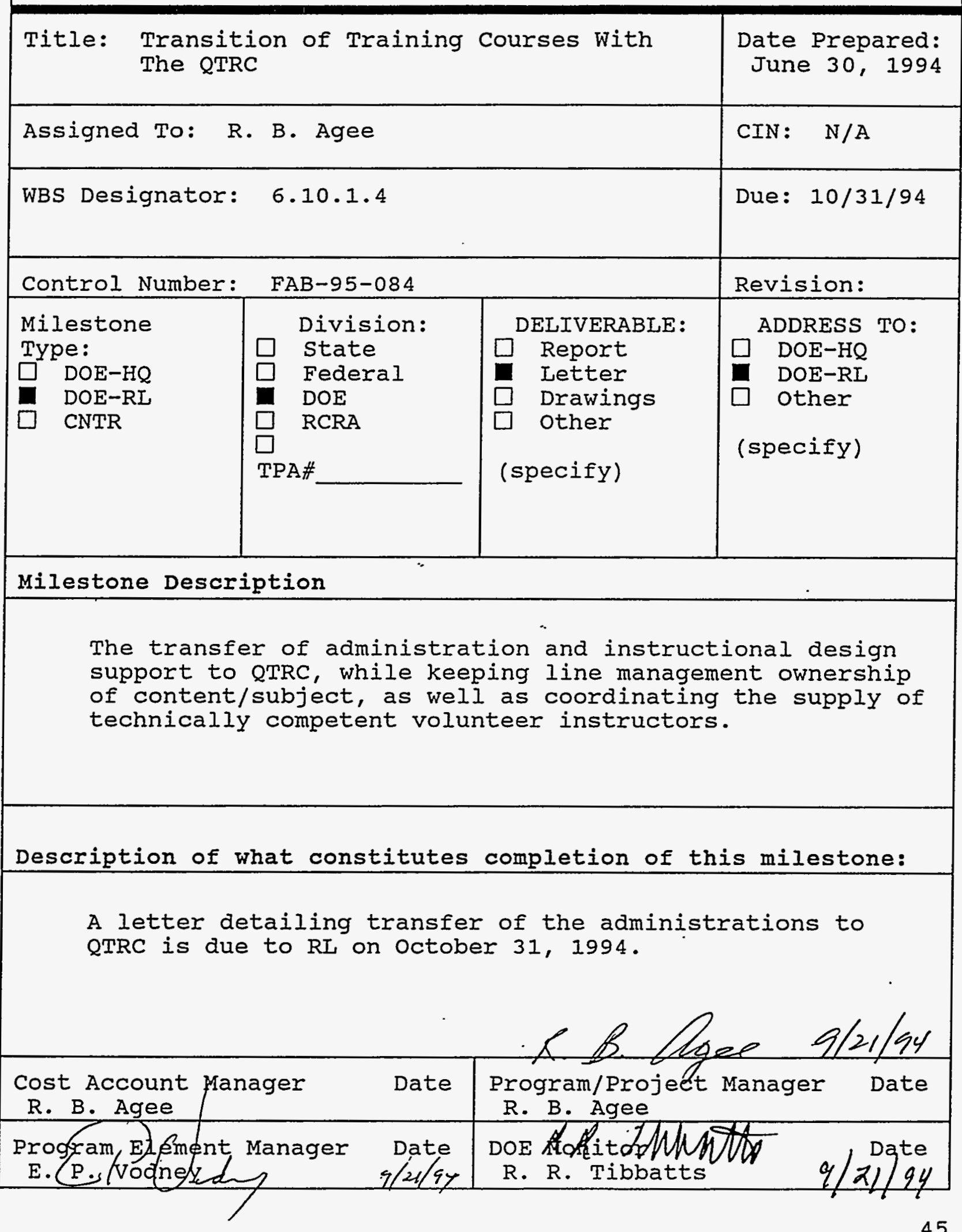


Title: Add Financial Curriculum courses to the Training Resource and Data Exchange electronic catalog

\begin{tabular}{|c|c|c|c|}
\hline Assigned To: $R$ & - B. Agee & & CIN: $\quad N / A$ \\
\hline WBS Designator: & 6.10 .1 .4 & & Due: $03 / 31 / 95$ \\
\hline Control Number: & $F A B-95-085$ & & Revision: \\
\hline $\begin{array}{l}\text { Milestone } \\
\text { Type: } \\
\square \text { DOE-HQ } \\
\text { DOE-RL } \\
\square \quad \text { CNTR }\end{array}$ & $\begin{array}{ll} & \text { Division: } \\
\square & \text { State } \\
\square & \text { Federal } \\
\text { DOE } \\
\square \text { RCRA } \\
\square \text { TPA\# }\end{array}$ & $\begin{array}{l}\text { DELIVERABLE: } \\
\square \text { Report } \\
\text { Letter } \\
\square \text { Drawings } \\
\square \text { other } \\
\text { (specify) }\end{array}$ & $\begin{array}{l}\text { ADDRESS TO: } \\
\square \text { DOE-HQ } \\
\text { DOE-RI } \\
\text { Other } \\
\text { (specify) }\end{array}$ \\
\hline \multicolumn{4}{|c|}{ Milestone Description } \\
\hline \multicolumn{4}{|c|}{$\begin{array}{l}\text { Inclusion of and coordinating the addition of all wEC } \\
\text { Government Contractor financial training courses. The } \\
\text { TRADE electronic course catalog is a course listing that } \\
\text { is available via bulletin board service to all DOE and its } \\
\text { contractors. The electronic catalog allows individuals } \\
\text { to access the contents from any site workstation. }\end{array}$} \\
\hline
\end{tabular}

Description of what constitutes completion of this milestone:

A letter detailing course catalog inclusion into the electronic BBS is due to RL on March 31, 1995.

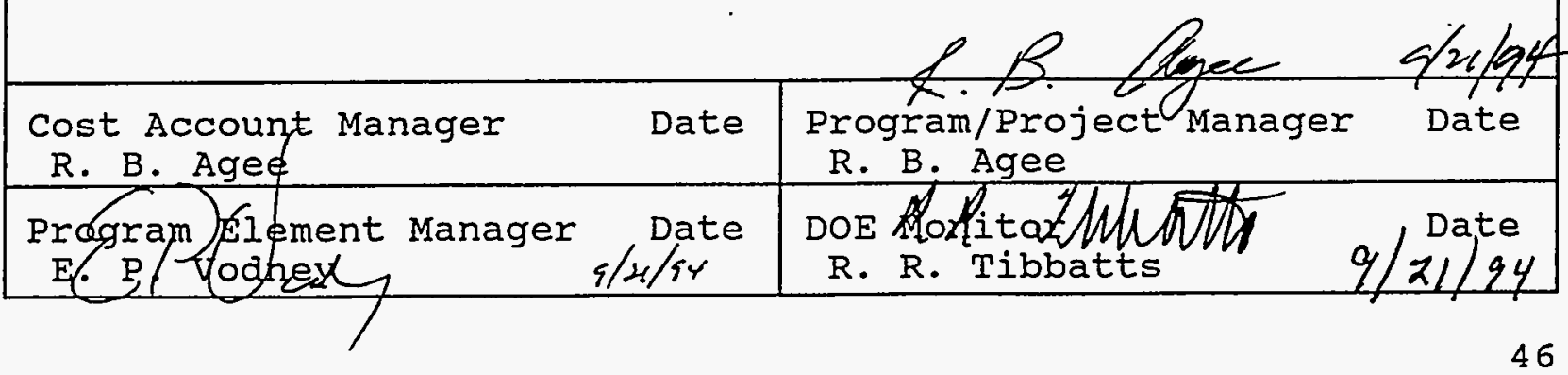

